RESEARCH ARTICLE

\title{
Global optimization of solar thermophotovoltaic systems
}

\author{
A. Datas and C. Algora
}

\begin{abstract}
In this paper, we present a theoretical model based on the detailed balance theory of solar thermophotovoltaic systems comprising multijunction photovoltaic cells, a sunlight concentrator and spectrally selective surfaces. The full system has been defined by means of $2 n+8$ variables (being $n$ the number of sub-cells of the multijunction cell). These variables are as follows: the sunlight concentration factor, the absorber cut-off energy, the emitter-to-absorber area ratio, the emitter cut-off energy, the band-gap energy(ies) and voltage(s) of the sub-cells, the reflectivity of the cells' back-side reflector, the emitter-to-cell and cell-to-cell view factors and the emitter-to-cell area ratio. We have used this model for carrying out a multi-variable system optimization by means of a multidimensional direct-search algorithm. This analysis allows to find the set of system variables whose combined effects results in the maximum overall system efficiency. From this analysis, we have seen that multijunction cells are excellent candidates to enhance the system efficiency and the electrical power density. Particularly, multijunction cells report great benefits for systems with a notable presence of optical losses, which are unavoidable in practical systems. Also, we have seen that the use of spectrally selective absorbers, rather than blackbody absorbers, allows to achieve higher system efficiencies for both lower concentration and lower emitter-to-absorber area ratio. Finally, we have seen that sun-to-electricity conversion efficiencies above $30 \%$ and electrical power densities above $50 \mathrm{~W} / \mathrm{cm}^{2}$ are achievable for this kind of systems.
\end{abstract}

\section{KEYWORDS}

solar thermophotovoltaic; detailed balance; energy balance; multijunction solar cells; sunlight concentration; spectral control; selective absorber

\section{INTRODUCTION}

Solar thermophotovoltaic (STPV) systems were proposed for the first time by Swanson in 1979 [1]. In these systems, an intermediate material is heated by the sun, re-radiating the absorbed energy towards a PV device (TPV cell). Then, either with the use of spectral filters between the cells and the emitter (the surface of the intermediate material facing the TPV cells) or with a special kind of spectrally selective emitters, the spectrum of the radiation impinging on the TPV cells is tuned to match the cell spectral response, and the non-useful photons for the photovoltaic conversion are turned back and re-absorbed by the emitter. The STPV technology has one of the highest solar-to-electricity ultimate conversion efficiency limit among the other photovoltaic approaches $(85.4 \%[2,3])$. Besides, the presence of an intermediate high temperature material allows to combine this technology with thermal storage systems and an alternate fuel/gas input [4,5]. Therefore, the main attractiveness of the STPV technology is that it could allow to build a high efficient, stable (non-intermittent), modular and scalable solution to generate electricity from the sun energy without using moving parts.

Unfortunately, the ultimate efficiency limit of an STPV system cannot be approached by practical systems. It is because of the many requirements imposed during the calculation of that efficiency. For instance, assuming maximum sunlight concentration factor (or alternatively, a special kind of angle-selective absorber), monochromatic exchange between the emitter and the cells, no nonradiative recombination neither ohmic losses at the cells, loss-free TPV optical cavity and a very large (approaching 
infinity) emitter-to-absorber area ratio, which would lead to a huge STPV system producing a negligible electrical power density (approaching zero).

Consequently, a less idealized (more complete) analysis is required to evaluate the real practical potentiality of STPV systems and more importantly to understand the practical requirements to achieve high system efficiency and electrical power density. Because of the inherent complexity of STPV system, most of the theoretical analysis that have been presented so far adopt several assumptions (maximum concentration factor, loss-free TPV cavity, a specific semiconductor material, etc.) to simplify the analytical problem [1,3,4,6-16]. However, because in an STPV system all the parameters are strongly correlated (affecting the equilibrium emitter temperature), the global optimization of these systems require a complex multi-variable analysis. In this regard, the first multivariable analysis of STPV systems was presented in [17], considering single junction TPV cells. In this paper, a more complete analysis is presented considering also multijunction TPV cells. More specifically, in this paper, we analyze an STPV system comprising an optical concentrator, a spectrally selective absorber, a spectrally selective emitter and multijunction TPV cells with an integrated back-side reflector (BSR). The analysis includes the effect of several variables on the system performance, such as the concentration factor, the emitter-to-absorber area ratio, the absorber cut-off energy, the semiconductor band-gap, the BSR reflectivity, the cavity optical losses, and so on

In addition to the optimization results presented in this paper, the mathematical formulation that has been developed can be applied to analyze STPV systems with different configurations.

\section{SYSTEM DESCRIPTION AND MODEL ASSUMPTIONS}

Figure 1 depicts the general scheme of the STPV system analyzed in this paper. In this system, the concentrated sunlight heats the absorber, which transfers that energy to the emitter surface with an arbitrarily larger area than that of the absorber. Then, the emitter radiates towards the TPV cells surrounding the emitter. We assume in an ideal case that both emitter and absorber temperatures are the same, meaning that an infinite thermal conductivity is connecting them. The radiation leaving the emitter surface is confined in the so-called TPV optical cavity. The design of such cavity must be optimized for the conversion of the emitter radiation into electricity at the TPV cells, turning back to the emitter the photons with energies below the band-gap of the TPV cells. On the other hand, the radiation leaving the absorber is lost.

In what follows, we review the particular properties of each component of this system: the sunlight receptor system, the TPV cells and the TPV optical cavity.

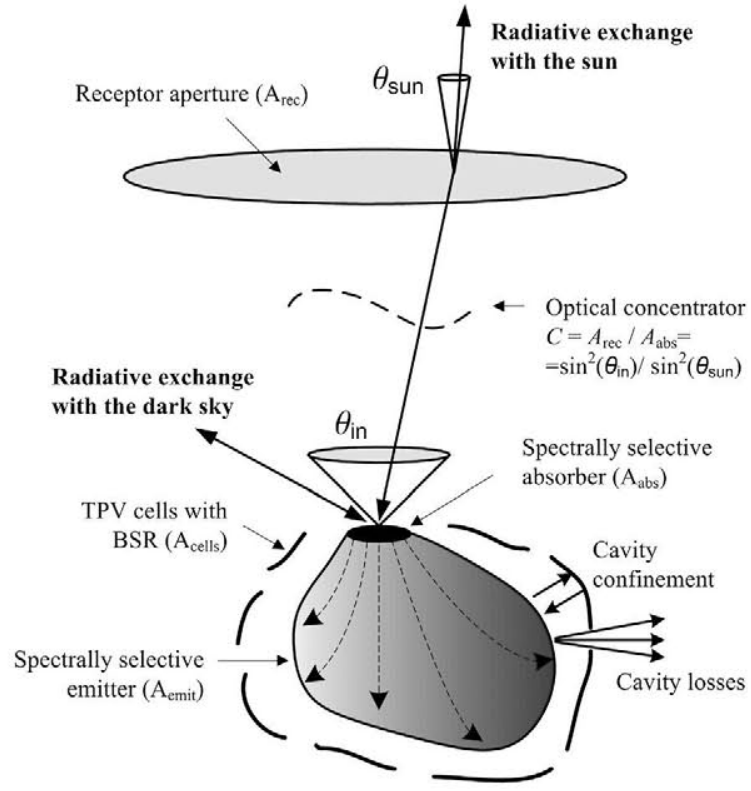

Figure 1. General scheme of a solar thermophotovoltaic (STPV) system.

\subsection{Sunlight receptor}

The sunlight receptor comprises the optical concentrator system and the absorber. We assume an ideal concentrator (without optical losses) able to concentrate the incident sunlight beam (impinging an area $A_{\text {rec }}$ and defining an angle $\left.\theta_{\text {sun }}\right)$ in an area $A_{\text {abs }}$ with an angle $\theta_{\text {in }}>\theta_{\text {sun }}$ (Figure 1). The $A_{\text {abs }}$ to $A_{\text {rec }}$ ratio is named concentration ratio $(C)$, and it can be formulated as a function of the beam angles $\theta_{\text {sun }}$ and $\theta_{\text {in }}$ :

$$
C=\frac{A_{\text {rec }}}{A_{\mathrm{abs}}}=\sin ^{2} \theta_{\text {in }} / \sin ^{2} \theta_{\mathrm{sun}}
$$

We also assume a diffuse spectrally selective absorber, with a cut-off energy $\varepsilon_{\mathrm{ca}}$, which only absorbs (and emits) the photons with energy above $\varepsilon_{\mathrm{ca}}$. Because of the overlap between the sun and the absorber spectra (Figure 2), the choice of $\varepsilon_{\text {ca }}$ must be determined by the trade-off between the emission and reflection losses at the absorber, which strongly depends on the absorber temperature and on the sunlight concentration ratio as it is illustrated in Figure 2. To simplify the analysis, an absorptivity of one is assumed for photon energies above $\varepsilon_{\mathrm{ca}}$ and zero for photon energies below $\varepsilon_{\mathrm{ca}}$.

Note that both the concentrator and the spectrally selective absorber are used to decrease the absorber emission losses: the former by reducing the absorber emission area and the latter by minimizing the spectral band of emission.

\subsection{TPV cells}

The TPV cells considered in this paper are monolithically series-connected multijunction cells (from now on, MJCs) 


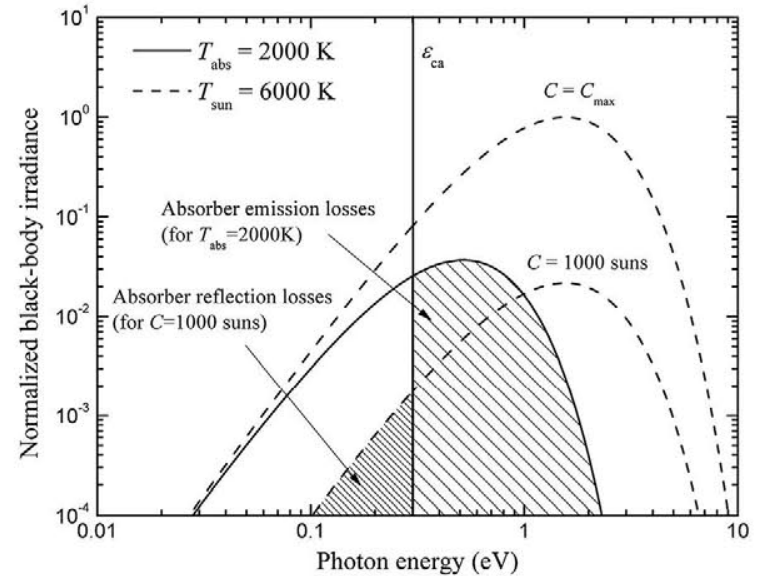

Figure 2. Spectral radiative exchange at the absorber for an arbitrary election of $\varepsilon_{\mathrm{ca}}=0.3 \mathrm{eV}, T_{\mathrm{abs}}=2000 \mathrm{~K}$ and $C=1000$ suns.

consisting of semiconductors with different band-gap energies $\varepsilon_{k}$, with $k$ ranging from 1 to $n$ (number of junctions). Figure 3 shows the band diagram of a dualjunction ( $n=2)$ TPV cell, and Figure 4 shows the radiative exchange within the cell. The front side of the cell is completely uncovered to allow the light to penetrate into the cell. In addition, the following assumptions are considered:

- Both $p$ and $n$ sides of each sub-cell are of the same semiconductor.

- The cells work in the radiative limit; that is, only radiative recombination takes place and each photon can only generate a single electron-hole pair (and vice versa) $[18,19]$.
- The sub-cells are series-connected by means of an ideal tunnel junction, which provides a non-resistive electrical contact between both sub-cells and can be assumed as transparent.

- The free electrons and holes have an infinite mobility within the semiconductor (zero resistance), so the quasi-Fermi level splitting for each sub-cell can be assumed constant and equal to $q V_{k}$ [19] (being $V_{k}$ the voltage at the $k$ th sub-cell).

- The cells are covered with an ideal anti-reflective coating; thus, all the photons impinging on the cell surface penetrate into the semiconductor.

- The cells have a specular BSR of reflectivity $\rho_{\mathrm{BSR}}$ (Figure 4).

- The photon-to-electron conversion takes place in certain regions of the semiconductor referred to as the sub-cell active layers (Figure 4), where all the photons with energies above the band-gap of the $\mathrm{k} t h$ cell $\left(\varepsilon_{k}\right)$ are absorbed and the rest are totally transmitted. In other words, the sub-cell spectral emissivity/absorptivity $\alpha_{\mathrm{d}, k}(\varepsilon)$ equals zero if $\varepsilon<\varepsilon_{k}$ and equals one otherwise.

Because no electrons are lost due to any mechanism besides radiative recombination, the current delivered to an external load can be directly calculated as the balance of the photon fluxes at the sub-cells. The flux of delivered electrons (electrical current) equals the balance between the absorbed photon flux (generation) and the emitted photon flux (radiative recombination). Note that in the case of series-connected MJCs, the voltage of each sub-cell is automatically tuned to provide the same current (electron generation rate minus electron recombination rate at each sub-cell). Moreover, the electron generation rate at each
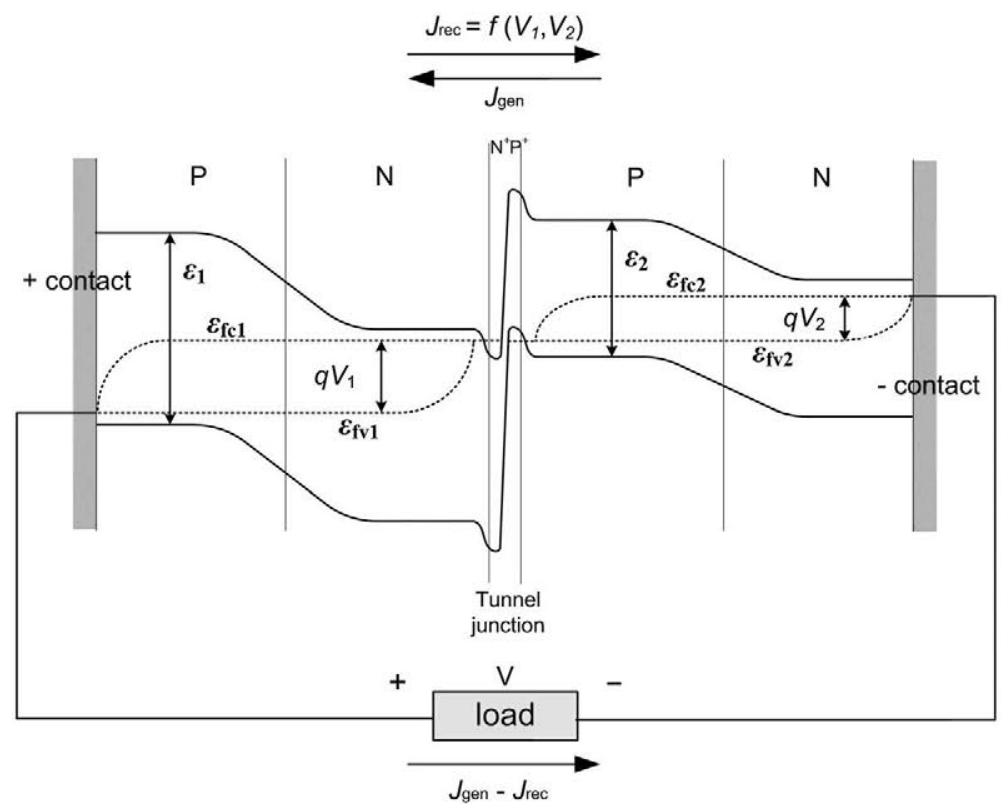

Figure 3. Band-gap diagram of an ideal illuminated dual-junction photovoltaic cell with band-gap energies $\varepsilon_{1}>\varepsilon_{2}$. 


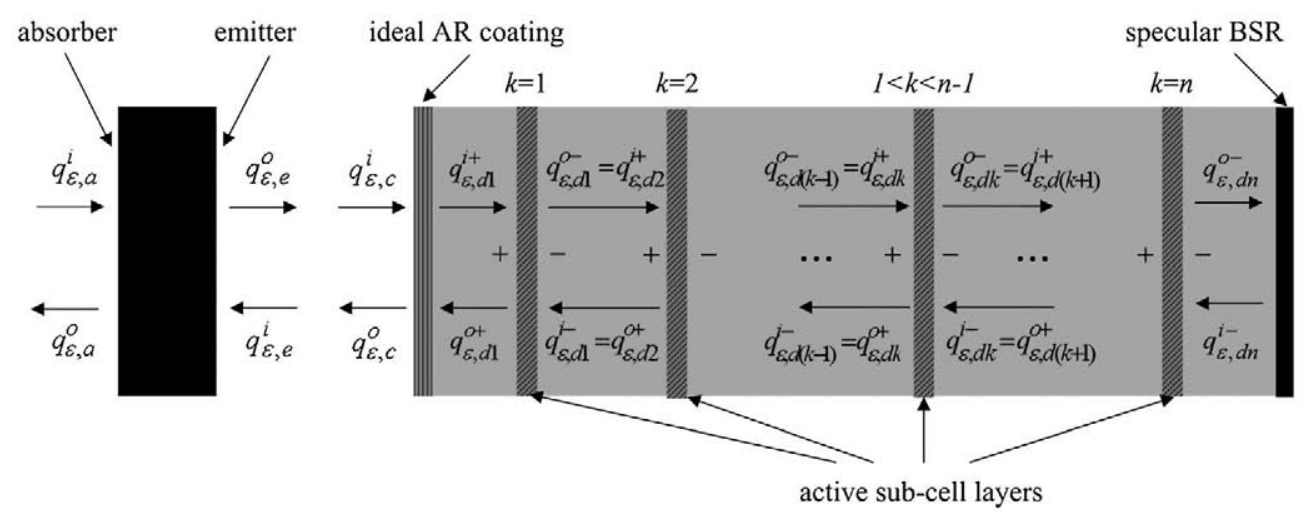

Figure 4. Radiation exchange within a solar thermophotovoltaic system using multijunction cell devices.

sub-cell is affected not only by the external irradiance, but also by the radiative recombination in other sub-cells, specifically those with higher band-gap energy. Thus, the generation rate at each sub-cell also depends on the voltage of the other sub-cells. Finally, the output voltage of the cell equals the sum of the quasi-Fermi levels splitting at each sub-cell (Figure 3).

\subsection{TPV optical cavity}

In a TPV system, the TPV cells enclose the emitter forming an optical cavity, which consists of the emitter, the cells and the inactive areas or open holes (Figure 1). In this paper, we assume that the emission from the emitter is restricted to photons with energies above $\varepsilon_{\mathrm{ce}}$, the emitter cut-off energy. In other words, the emitter spectral emissivity/absorptivity $\alpha_{\mathrm{e}}(\varepsilon)$ is zero if $\varepsilon<\varepsilon_{\mathrm{ce}}$ and one otherwise. For this cavity, we assume that

- Every surface (except the BSR) emits and reflects in a diffuse manner.

- The BSR is a perfectly specular reflector with reflectivity $\rho_{\mathrm{BSR}}$.

- All the surfaces are isothermal.

- The emission originating from surfaces at room temperature and with $\mu=0$ is negligible. This refers to the BSR and inactive areas within the cavity.

- The emitter is convex (it cannot see itself) and opaque.

The first assumption allows us to simplify the cavity analysis because the fraction of the energy flux leaving a surface $x$ that impinges a surface $y$ when surface $z$ is a shadowing obstacle can be expressed by a purely geometrical factor, the view factor $F_{x y}^{(z)}$ [20].

\section{MODEL FORMULATION}

The total photon flux and energy flux ( $N$ and $E$, respectively) of photons with electrochemical potential $\mu$ and energy between $\varepsilon_{1}$ and $\varepsilon_{2}$ that are emitted by a surface $\mathrm{d} A$ at temperature $T$ immersed in a medium of refraction index $n_{\text {out }}$ in a solid angle $\mathrm{d} \Omega$ and in a direction defined by an angle $\theta$ with the surface normal, are given by the generalized Planck equation [2,21]:

$$
\begin{array}{r}
N=\int_{H} \dot{N}\left(\varepsilon_{1}, \varepsilon_{2}, T, \mu\right) \mathrm{d} H \equiv \int_{H} \int_{\varepsilon_{1}}^{\varepsilon_{2}} e(\varepsilon, \theta, \varphi) \cdot \dot{n}(\varepsilon, T, \mu) \mathrm{d} \varepsilon \mathrm{d} H \\
\equiv \frac{2}{h^{3} c^{2}} \int_{H} \int_{\varepsilon_{1}}^{\varepsilon_{2}} e(\varepsilon, \theta, \varphi) \cdot \frac{\varepsilon^{2} \mathrm{~d} \varepsilon \mathrm{d} H}{\exp \left(\frac{\varepsilon-\mu}{k T}\right)-1}
\end{array}
$$

$$
\begin{array}{r}
E=\int_{H} \dot{E}\left(\varepsilon_{1}, \varepsilon_{2}, T, \mu\right) \mathrm{d} H=\int_{H} \int_{\varepsilon_{1}}^{\varepsilon_{2}} e(\varepsilon, \theta, \varphi) \cdot \dot{e}(\varepsilon, T, \mu) \mathrm{d} \varepsilon \mathrm{d} H \\
\equiv \frac{2}{h^{3} c^{2}} \int_{H} \int_{\varepsilon_{1}}^{\varepsilon_{2}} e(\varepsilon, \theta, \varphi) \cdot \frac{\varepsilon^{3} \mathrm{~d} \varepsilon \mathrm{d} H}{\exp \left(\frac{\varepsilon-\mu}{k T}\right)-1}
\end{array}
$$

where $h$ is Planck's constant, $k$ is Boltzmann's constant, $c$ is the speed of light in a vacuum, $e(\varepsilon, \theta, \varphi)$ is the spectraldirectional emissivity of the surface, $\dot{n}(\varepsilon, T, \mu)$ and $\dot{e}(\varepsilon, T, \mu)$ denote the spectral photon and energy fluxes emitted in vacuum in the normal direction and per unit of solid angle, and $\dot{N}\left(\varepsilon_{1}, \varepsilon_{2}, T, \mu\right)$ and $\dot{E}\left(\varepsilon_{1}, \varepsilon_{2}, T, u\right)$ denote the total photon and the energy fluxes emitted in vacuum, in the spectral interval $\left(\varepsilon_{1}, \varepsilon_{2}\right)$, in the normal direction and per unit of solid angle. $d H$ is the differential of the Lagrange invariant or étendue (extension) given by $\mathrm{d} H \equiv n_{\text {out }}^{2} \cos \theta \mathrm{d} A \mathrm{~d} \Omega=$ $n_{\text {out }}^{2} \cos \theta \sin \theta \mathrm{d} A \mathrm{~d} \theta \mathrm{d} \varphi[22]$.

In this paper, it is assumed that $e(\varepsilon, \theta, \varphi)=1$ for the interval $\left(\varepsilon_{1}-\varepsilon_{2}\right)$ and for every $\theta$ and $\varphi$. Therefore, the integration over spatial/angular coordinates can be separated from the integration in photon energies, resulting that

$$
N=H \cdot \dot{N}\left(\varepsilon_{1}, \varepsilon_{2}, T, \mu\right)
$$

$$
E=H \cdot \dot{E}\left(\varepsilon_{1}, \varepsilon_{2}, T, \mu\right)
$$

where $H$ is the integral of $d H$ over $A,\left(\theta_{\min }, \theta_{\max }\right)$ and $\left(\varphi_{\min }, \varphi_{\max }\right)$ : 


$$
\begin{aligned}
H & =n_{\text {out }}^{2} \cdot \int_{A} \int_{\theta_{\min }}^{\theta_{\max }} \int_{\varphi_{\min }}^{\varphi_{\max }} \cos \theta \sin \theta \mathrm{d} A \mathrm{~d} \theta \mathrm{d} \varphi= \\
& =\frac{n_{\text {out }}^{2}}{2} \cdot A \cdot\left(\varphi_{\max }-\varphi_{\min }\right) \cdot\left(\sin ^{2} \theta_{\max }-\sin ^{2} \theta_{\min }\right)
\end{aligned}
$$

In geometrical optics, $H$ represents the volume that a beam fills in the phase-space domain [22]; thus, it represents the spatial/angular extension of emission, and it must be conserved in a non-lossy optical system.

Therefore, in this paper, the emission from every surface in an STPV system is described by means of five parameters: $H, \varepsilon_{1}, \varepsilon_{2}, T$ and $\mu$ (Equations (2)-(3)), which depend on the nature of the radiation source. Table I summarizes these parameters for the four sources existing in an STPV system, that is, the sun, the absorber, the emitter and the TPV cells.

The radiative energy fluxes that exist in the STPV system are shown in Figure 4. The spectral energy flux impinging the + side of a surface $x$ is denoted as $q_{\varepsilon, x}^{\mathbf{i}+}$, whereas the spectral energy flux outgoing the - side of a surface $x$ is denoted as $q_{\varepsilon, x}^{0-}$.

The light impinging the absorber $q_{\varepsilon, \mathrm{a}}^{\mathrm{i}}$ heats the absorber/ emitter component increasing its temperature. Consequently, it radiates from both the absorber $\left(q_{\varepsilon, \mathrm{a}}^{\mathrm{o}}\right)$ and the emitter $\left(q_{\varepsilon, \mathrm{e}}^{\mathrm{o}}\right)$ sides. Part of the emitted radiation impinges the cells $\left(q_{\varepsilon, \mathrm{c}}^{\mathbf{i}}\right)$ and penetrates into the semiconductor, illuminating the top cell $\left(q_{\varepsilon, \mathrm{d} 1}^{\mathrm{i}+}\right)$. The photons with energies below the band-gap of the top cell $\left(\varepsilon_{1}\right)$ pass through this cell and illuminate the next one, and so on. Finally, the part of the spectrum that was not absorbed by any cell $\left(q_{\varepsilon, \mathrm{d} n}^{\mathrm{o}}\right)$ is reflected in the BSR $\left(q_{\varepsilon, \mathrm{d} n}^{\mathrm{i}-}\right)$ and is turned back. At the same time, when a sub-cell is forward biased, it emits photons with chemical potential $\mu=q V_{k}$ (luminescent radiation [21]) from both sides ( + and - ) of its active layer, contributing to the energy fluxes outgoing from these layers $\left(q_{\varepsilon, \mathrm{d} k}^{\mathrm{o}-}\right.$ and $\left.q_{\varepsilon, \mathrm{d} k}^{\mathrm{o}+}\right)$. Part of these fluxes is absorbed by the adjacent cells, and another part comes out of the cell $\left(q_{\varepsilon, c}^{\mathrm{o}}\right)$ and illuminates the emitter surface $\left(q_{\varepsilon, \mathrm{e}}^{\mathrm{i}}\right)$. The net radiation method [20] is used to calculate all these fluxes. The fluxes inside the cells (Figure 4) are obtained by solving the following system of $4 n$ equations ( $k$ goes from 1 to $n$ ):

$$
\begin{aligned}
q_{\varepsilon, \mathrm{d} k}^{\mathrm{o}}(\varepsilon)= & \pi n_{\mathrm{int}}^{2} \alpha_{\mathrm{d} k}(\varepsilon) \cdot \dot{e}\left(\varepsilon, T_{\text {subcell }}, q V_{k}\right) \\
& +\left(1-\alpha_{\mathrm{d} k}(\varepsilon)\right) \cdot q_{\varepsilon, \mathrm{d} k}^{\mathrm{i}+}(\varepsilon)
\end{aligned}
$$

$$
\begin{aligned}
& q_{\varepsilon, \mathrm{d} k}^{\mathrm{o}+}(\varepsilon)= \pi n_{\mathrm{int}}^{2} \alpha_{\mathrm{d} k}(\varepsilon) \cdot \dot{e}\left(\varepsilon, T_{\text {subcel } \left.1, q V_{k}\right)}\right. \\
&+\left(1-\alpha_{\mathrm{d} k}(\varepsilon)\right) \cdot q_{\varepsilon, \mathrm{d} k}^{\mathrm{i}-}(\varepsilon) \\
& q_{\varepsilon, \mathrm{d} k}^{\mathrm{i}+}(\varepsilon)=q_{\varepsilon, \mathrm{d}(k-1)}^{\mathrm{o}}(\varepsilon) \\
& q_{\varepsilon, \mathrm{d} k}^{\mathrm{i}-}(\varepsilon)=q_{\varepsilon, \mathrm{d}(k+1)}^{\mathrm{o}+}(\varepsilon)
\end{aligned}
$$

where $\dot{e}(\varepsilon, T, \mu)$ is given by Equation (3) and $n_{\text {int }}$ is the refractive index of the semiconductor, which is around 3.5 for most of the $\mathrm{III}-\mathrm{V}$ semiconductors commonly used to manufacture MJCs. To solve the system of Equations (7)-(10), we must note two exceptions to the general formulation shown in this equations: First, it should be referred that the + side of the top cell $(k=1)$ is not only illuminated by the incident radiation $\left(q_{\varepsilon, c}^{\mathrm{i}}(\varepsilon)\right)$ but also by the internal total reflection at the semiconductor/air interface of the luminescent emission originated at the top cell $(k=1)$. In this regard, note that the fraction of the incident radiation, which is reflected at the specular BSR is not internally reflected at the semiconductor/air interface, but is directly transmitted out of the cell as isotropic radiation. Therefore, we can write

$$
\begin{aligned}
q_{\varepsilon, \mathrm{d} 1}^{\mathrm{i}+}(\varepsilon)= & q_{\varepsilon, \mathrm{c}}^{\mathrm{i}}(\varepsilon)+\left(1-1 / n_{\mathrm{int}}^{2}\right) \cdot \\
& {\left[q_{\varepsilon, \mathrm{d} 1}^{\mathrm{o}+}(\varepsilon)-\rho_{\mathrm{BSR}} \cdot\left(1-\alpha_{\mathrm{d} n}(\varepsilon)\right) \cdot q_{\varepsilon, \mathrm{c}}^{\mathrm{i}}(\varepsilon)\right] }
\end{aligned}
$$

The second exception is related with the bottom-cell $(k=n)$, for which we must write

$$
q_{\varepsilon, \mathrm{d} n}^{\mathbf{i}-}(\varepsilon)=\rho_{\mathrm{BSR}} \cdot q_{\varepsilon, \mathrm{d} n}^{\mathrm{o}}(\varepsilon)
$$

The fluxes obtained from Equations (7)-(12), that is, $q_{\varepsilon, \mathrm{d} k}^{\mathrm{i}+}(\varepsilon), q_{\varepsilon, \mathrm{d} k}^{\mathrm{i}-}(\varepsilon), q_{\varepsilon, \mathrm{d} k}^{\mathrm{o}-}(\varepsilon)$ and $q_{\varepsilon, \mathrm{d} k}^{\mathrm{o}+}(\varepsilon)$ can be expressed as a function of the energy flux impinging the cells surface, $q_{\varepsilon, \mathrm{c}}^{\mathrm{i}}(\varepsilon)$. Besides, for the emitter and the outside boundary of the cells (or anti-reflective coating) we can write

$$
\begin{gathered}
q_{\varepsilon, \mathrm{e}}^{\mathrm{o}}(\varepsilon)=\pi \alpha_{\mathrm{e}}(\varepsilon) \cdot \dot{e}\left(\varepsilon, T_{\mathrm{emit}}, 0\right)+\left(1-\alpha_{\mathrm{e}}(\varepsilon)\right) \cdot q_{\varepsilon, \mathrm{e}}^{\mathrm{i}}(\varepsilon) \\
q_{\varepsilon, \mathrm{c}}^{\mathrm{o}}(\varepsilon)=\left(1 / n_{\mathrm{int}}^{2}\right) \cdot\left[q_{\varepsilon, \mathrm{d} 1}^{\mathrm{o}+}(\varepsilon)-\rho_{\mathrm{BSR}} \cdot\left(1-\alpha_{\mathrm{d} n}(\varepsilon)\right) \cdot q_{\varepsilon, \mathrm{c}}^{\mathrm{i}}(\varepsilon)\right]+ \\
+\rho_{\mathrm{BSR}} \cdot\left(1-\alpha_{\mathrm{d} n}(\varepsilon)\right) \cdot q_{\varepsilon, \mathrm{c}}^{\mathrm{i}}(\varepsilon) \\
q_{\varepsilon, \mathrm{e}}^{\mathrm{i}}(\varepsilon)=F_{\mathrm{ec}} \cdot q_{\varepsilon, \mathrm{c}}^{\mathrm{o}}(\varepsilon) \\
q_{\varepsilon, \mathrm{c}}^{\mathrm{i}}(\varepsilon)=F_{\mathrm{ce}} \cdot q_{\varepsilon, \mathrm{e}}^{\mathrm{o}}(\varepsilon)+F_{\mathrm{cc}}^{(\mathrm{e})} \cdot q_{\varepsilon, \mathrm{c}}^{\mathrm{o}}(\varepsilon)
\end{gathered}
$$

Table I. Parameters defining the radiation originating from each component of an idealized solar thermophotovoltaic (STPV) system.

\begin{tabular}{llllll}
\hline Source & \multicolumn{1}{c}{$H$} & $\varepsilon_{1}$ & $\varepsilon_{2}$ & $T$ & $\mu$ \\
\hline Sun & $\pi A_{\text {rec }} \sin ^{2} \theta_{\text {sun }}=\pi A_{\text {abs }} \sin ^{2} \theta_{\text {in }}$ & 0 & $\infty$ & 6000 & 0 \\
Absorber & $\pi A_{\text {abs }}$ & $\varepsilon_{c a}$ & $\infty$ & $T_{\text {emit }}$ & 0 \\
Emitter & $\pi A_{\text {emit }}$ & $\varepsilon_{c e}$ & $\infty$ & $T_{\text {emit }}$ & 0 \\
TPV sub-cells & $\pi A_{\text {cells }} n_{\text {int }}^{2}$ & $\varepsilon_{k}>\varepsilon_{k+1} \geq \varepsilon_{c e}$ & $\infty$ & 300 & $q V_{k}$ \\
\hline
\end{tabular}


The fluxes obtained from Equations (13)-(16), that is, $q_{\varepsilon, \mathrm{c}}^{\mathrm{i}}(\varepsilon), q_{\varepsilon, \mathrm{e}}^{\mathrm{i}}(\varepsilon), q_{\varepsilon, \mathrm{e}}^{\mathrm{o}}(\varepsilon)$ and $q_{\varepsilon, \mathrm{c}}^{\mathrm{o}}(\varepsilon)$, are then obtained as a function of $q_{\varepsilon, \mathrm{d} 1}^{\mathrm{o}+}(\varepsilon)$. Hence, the combination of Equations (7)-(16) provides a close solution for all the fluxes between the emitter and the cells. Finally, for the absorber, we have

$$
\begin{aligned}
q_{\varepsilon, \mathrm{a}}^{\mathrm{o}}(\varepsilon)= & \pi \alpha_{\mathrm{a}}(\varepsilon) \cdot \dot{e}\left(\varepsilon, T_{\mathrm{emit}}, 0\right)+\left(1-\alpha_{\mathrm{a}}(\varepsilon)\right) \cdot q_{\varepsilon, \mathrm{a}}^{i}(\varepsilon) \\
q_{\varepsilon, \mathrm{a}}^{\mathrm{i}}(\varepsilon)= & \pi\left(C / C_{\max }\right) \cdot \dot{e}\left(\varepsilon, T_{\mathrm{sun}}, 0\right) \\
& +\pi\left(1-C / C_{\max }\right) \cdot \dot{e}\left(\varepsilon, T_{\mathrm{sky}}, 0\right)
\end{aligned}
$$

where $T_{\text {sky }}$ is the temperature of the dark parts of the sky, excluding the sun disc, assumed to be $300 \mathrm{~K}$. These equations provide a close solution for the spectral energy fluxes at the absorber, that is, $q_{\varepsilon, \mathrm{a}}^{\mathrm{o}}(\varepsilon)$ and $q_{\varepsilon, \mathrm{a}}^{\mathrm{i}}(\varepsilon)$. It is important to refer that all the fluxes are dependent on the emitter temperature $T_{\text {emit }}$, which results from the following energy balance in the emitter/absorber:

$$
\begin{array}{r}
\int_{0}^{\infty}\left[A_{\mathrm{abs}} \cdot\left(q_{\varepsilon, \mathrm{a}}^{i}(\varepsilon)-q_{\varepsilon, \mathrm{a}}^{o}(\varepsilon)\right)+A_{\text {emit }} \cdot\right. \\
\left.\left(q_{\varepsilon, \mathrm{e}}^{i}(\varepsilon)-q_{\varepsilon, \mathrm{e}}^{o}(\varepsilon)\right)\right] \mathrm{d} \varepsilon=0
\end{array}
$$

Introducing the solution of Equations (7) (18) into Equation (19), we finally obtain

$$
\begin{aligned}
\frac{C}{C_{\mathrm{max}}} \cdot & {\left[\dot{E}\left(\varepsilon_{\mathrm{ca}}, \infty, T_{\mathrm{sun}}, 0\right)-\dot{E}\left(\varepsilon_{\mathrm{ca}}, \infty, T_{\mathrm{emit}}, 0\right)\right]+} \\
& +\left(1-\frac{C}{C_{\mathrm{max}}}\right) \cdot\left[\dot{E}\left(\varepsilon_{\mathrm{ca}}, \infty, T_{\mathrm{sky}}, 0\right)-\dot{E}\left(\varepsilon_{\mathrm{ca}}, \infty, T_{\mathrm{emit}}, 0\right)\right]+ \\
& +\left(\frac{A_{\mathrm{emit}}}{A_{\mathrm{abs}}}\right) \cdot\left[F_{\mathrm{ec}} \cdot \sum_{k=1}^{n} \dot{E}\left(\varepsilon_{k}, \varepsilon_{\mathrm{c}-1}, T_{\mathrm{cells}}, q V_{k}\right)-\dot{E}\left(\varepsilon_{\mathrm{ce}}, \infty, T_{\mathrm{emit}}, 0\right)+\right. \\
& \left.+\frac{A_{\mathrm{emit}}}{A_{\mathrm{cells}}} \cdot \frac{\rho_{\mathrm{BSR}} F_{\mathrm{ec}}^{2}}{1-\rho_{\mathrm{BSR}} F_{\mathrm{cc}}^{(\mathrm{e})}} \cdot \dot{E}\left(\varepsilon_{\mathrm{ce}}, \varepsilon_{n}, T_{\mathrm{emit}}, 0\right)\right]=0
\end{aligned}
$$

where $\dot{E}\left(\varepsilon_{1}, \varepsilon_{2}, T, \mu\right)$ is given by Equation (3), $C$ is the sunlight concentration factor (Equation (1)), $C_{\max }=1 / \sin ^{2} \theta_{\text {sun }}$ is the maximum concentration factor achievable on the earth surface (assuming a material with a refractive index of one surrounding the absorber), and $F_{\mathrm{ec}}$ and $F_{\mathrm{cc}}^{(\mathrm{e})}$ are the emitterto-cells and cells-to-cells view factors, respectively.

The first term in Equation (20) accounts for the radiation exchange between the sun and the absorber, which is limited to photons with $\varepsilon>\varepsilon_{\mathrm{ca}}$ and within a cone forming an angle $\theta_{\text {in }}$ with respect to the surface normal ( $\theta_{\text {in }}=$ $\arcsin \sqrt{C / C_{\max }}$ ) (Figure 1). The second term corresponds to the radiation exchange between the absorber and the dark part of the sky, which usually represents losses, as long as $T_{\text {sky }}<T_{\text {emit }}$. The third term represents the energy balance at the emitter surface, which depends on the optical cavity that encloses the emitter. Within this term, the first term $\left(F_{\text {ec }} \cdot \sum_{k=1}^{n} \dot{E}\left(\varepsilon_{k}, \varepsilon_{k-1}, T_{\text {cells }}, q V_{k}\right)\right)$ represents the luminescent radiation originated at the different sub-cells due to radiative recombination that is absorbed by the emitter. Note that for the first term of the summation, $\varepsilon_{k-1}=\varepsilon_{0} \rightarrow \infty$. The second one $\left(\dot{E}\left(\varepsilon_{\mathrm{ce}}, \infty, T_{\text {emit }}, 0\right)\right)$ represents the total energy flux radiated by the emitter, and the last term
$\left(\frac{A_{\mathrm{emit}}}{A_{\mathrm{cells}}} \cdot \frac{\rho_{\mathrm{BSR}} F_{\mathrm{ec}}^{2}}{1-\rho_{\mathrm{BSR}} F_{\mathrm{cc}}^{(\theta)}} \cdot \dot{E}\left(\varepsilon_{\mathrm{ce}}, \varepsilon_{n}, T_{\mathrm{emit}}, 0\right)\right)$ represents the portion of the emitted radiation that is turned back by the TPV cavity and finally re-absorbed by the emitter. The solution of Equation (20) provides the equilibrium emitter temperature $\left(T_{\text {emit }}\right)$, as long as $T_{\text {sun }}=6000 \mathrm{~K}$ and $T_{\text {sky }}=T_{\text {env }}=T_{\text {cells }}=$ $300 \mathrm{~K}$ are known.

Once the equilibrium emitter temperature is known, the current generated by the TPV cells can be calculated. In the radiative limit, the net rate of absorbed photons in each sub-cell equals the net rate of generated electrons, which determines the electrical current density:

$J_{k}=\frac{q}{A_{\text {cells }}}$. [incoming photon flux - outgoing photon flux $]=$ $=q \cdot \int_{\varepsilon_{k}}^{\infty} \frac{1}{\varepsilon} \cdot\left[q_{\varepsilon, \mathrm{d} k}^{i+}(\varepsilon)+q_{\varepsilon, \mathrm{d} k}^{i-}(\varepsilon)-q_{\varepsilon, \mathrm{d} k}^{o+}(\varepsilon)-q_{\varepsilon, \mathrm{d} k}^{o-}(\varepsilon)\right] \mathrm{d} \varepsilon$

The photocurrent density generated in each sub-cell $J_{k}$ is obtained from the solution of the radiative energy fluxes (Equations (7)-(18)):

$$
\begin{aligned}
& J_{1} / q= \pi\left[\left(A_{\mathrm{emit}} / A_{\mathrm{cells}}\right) \cdot F_{\mathrm{ec}} \cdot \dot{N}\left(\varepsilon_{1}, \infty, T_{\mathrm{emit}}, 0\right)\right. \\
&-\left.\left(1-F_{\mathrm{cc}}^{(\mathrm{e})}\right) \cdot \dot{N}\left(\varepsilon_{1}, \infty, T_{\mathrm{cells}}, q V_{1}\right)\right]+\pi n_{\mathrm{int}}^{2} \\
& \cdot\left[\dot{N}\left(\varepsilon_{1}, \infty, T_{\mathrm{cells}}, q V_{2}\right)-\dot{N}\left(\varepsilon_{1}, \infty, T_{\mathrm{cells}}, q V_{1}\right)\right] \\
& J_{n} / q=\pi\left[\left(A_{\mathrm{emit}} / A_{\mathrm{cells}}\right) \cdot F_{\mathrm{ec}} \cdot \dot{N}\left(\varepsilon_{n}, \varepsilon_{n-1}, T_{\mathrm{emit}}, 0\right)\right. \\
&\left.-\left(1-F_{\mathrm{cc}}^{(\mathrm{e})}\right) \cdot \dot{N}\left(\varepsilon_{n}, \varepsilon_{n-1}, T_{\mathrm{cells}}, q V_{n}\right)\right]+ \\
&+\pi n_{\mathrm{int}}^{2} \cdot\left[\dot{N}\left(\varepsilon_{n-1}, \infty, T_{\mathrm{cells}}, q V_{n-1}\right)\right. \\
&-\dot{N}\left(\varepsilon_{n-1}, \infty, T_{\mathrm{cells}}, q V_{n}\right)- \\
&\left.-\left(1-\rho_{\mathrm{BSR}}\right) \cdot \dot{N}\left(\varepsilon_{n}, \infty, T_{\mathrm{cells}}, q V_{n}\right)\right]
\end{aligned}
$$

$$
\begin{aligned}
J_{k} / q= & \pi\left[\left(A_{\mathrm{emit}} / A_{\mathrm{cells}}\right) \cdot F_{\mathrm{ec}} \cdot \dot{N}\left(\varepsilon_{k}, \varepsilon_{k-1}, T_{\mathrm{emit}}, 0\right)\right. \\
& \left.-\left(1-F_{\mathrm{cc}}^{(\mathrm{e})}\right) \cdot \dot{N}\left(\varepsilon_{k}, \varepsilon_{k-1}, T_{\mathrm{d}}, q V_{k}\right)\right]+ \\
& +\pi n_{\mathrm{int}}^{2} \cdot\left[\dot{N}\left(\varepsilon_{k-1}, \infty, T_{\mathrm{cells}}, q V_{k-1}\right)\right. \\
& +\dot{N}\left(\varepsilon_{k}, \infty, T_{\mathrm{cells}}, q V_{k+1}\right)- \\
& \left.-\dot{N}\left(\varepsilon_{k}, \infty, T_{\text {cells }}, q V_{k}\right)-\dot{N}\left(\varepsilon_{k-1}, \infty, T_{\text {cells }}, q V_{k}\right)\right]
\end{aligned}
$$

Equation (22) gives the current density of the top cell, Equation (23) the current density of the bottom-cell and Equation (24) the current density of an intermediate subcell, being $1<k<n$. In these equations, the positive terms account for the net generation rate of electron-hole 
pairs, and the negative ones account for the net radiative recombination rate from both the + side and - side of the sub-cell active layers. Besides, because the sub-cells are connected in series, they must deliver the same current, resulting in $n-1$ additional conditions:

$$
J_{k}=J_{k+1} \quad \forall k \text { from } 1 \text { to } n-1
$$

Lastly, the electrical power density (watts per unit of TPV cell area) and the STPV system efficiency, defined as the ratio between electrical and incident sun power, are given by

$$
\begin{gathered}
P_{\mathrm{d}}=J \cdot \sum_{k=1}^{n} V_{k} \\
\eta_{\mathrm{STPV}}=\frac{A_{\text {cells }} \cdot P_{\mathrm{d}}}{A_{\text {rec }} \sin ^{2}\left(\theta_{\mathrm{sun}}\right) \cdot \sigma T_{\mathrm{sun}}^{4}} \\
=\frac{A_{\text {cells }}}{A_{\text {emit }}} \cdot \frac{A_{\mathrm{emit}}}{A_{\mathrm{abs}}} \cdot \frac{C_{\mathrm{max}}}{C} \cdot \frac{P_{\mathrm{d}}}{\sigma T_{\text {sun }}^{4}}
\end{gathered}
$$

where $\sigma$ is the Stefan-Boltzmann constant and $J=J_{k}$ for all $n \leq k \leq 1$, as stated by Equation (25).

\section{CALCULATION METHOD}

To find the equilibrium emitter temperature requires the simultaneous solution of Equations (20) and (25), resulting in a total of $n$ equations that can be solved for $n$ unknown system parameters. In this study, the unknown parameters are taken to be the emitter temperature $T_{\text {emit }}$ and the voltage of the $n-1$ first sub-cells, that is, $V_{k}$ with $k$ ranging from 1 to $n-1$. The rest of the variables in the $n$ equations must be fixed to find a unique solution for the system of equations. These variables are the following (Table II): voltage of the $n$th sub-cell $\left(V_{n}\right)$, band-gap energy of all the subcells ( $\varepsilon_{k}$, for $k$ ranging from 1 to $n$ ), concentration factor $(C$ ), absorber cut-off energy $\left(\varepsilon_{\mathrm{ca}}\right)$, emitter cut-off energy $\left(\varepsilon_{\mathrm{ce}}\right)$, emitter-to-absorber area ratio $\left(A_{\text {emit }} / A_{\text {abs }}\right)$, emitter-to-cells area ratio $\left(A_{\text {emit }} / A_{\text {cells }}\right)$, BSR reflectivity $\left(\rho_{\mathrm{BSR}}\right)$, emitterto-cells view factor $\left(F_{\mathrm{ec}}\right)$ and cells-to-cells view factor
$\left(F_{\mathrm{cc}}^{(\mathrm{e})}\right)$. They represent a total of $n+9$ variables, which make the analysis of this kind of systems very complex.

In this paper, the following assumptions are held: blackbody emitter $\left(\varepsilon_{\mathrm{ce}}=0\right)$ that the cells do not see each other $\left(F_{\mathrm{cc}}^{(\mathrm{e})}=0\right)$ and that the cells only see the emitter $\left(F_{\mathrm{ce}}=1\right.$ or equivalently $\left.F_{\text {ec }}=A_{\text {cells }} / A_{\text {emit }}\right)$. These conditions eliminate three variables, remaining $n+6$ free parameters, which are as follows: $C, V_{n}, \varepsilon_{k}, \varepsilon_{\mathrm{ca}}, A_{\mathrm{emit}} / A_{\mathrm{abs}}, F_{\mathrm{ec}}$ and $\rho_{\mathrm{BSR}}$. Further assumptions are considered along the paper to simplify the analysis, and they are pointed out as they emerge.

Finally, in this paper, a full system optimization is performed, which attempts to find the optimum set of parameters that maximize the system conversion efficiency. For that, a multidimensional direct-search Nelder-Mead algorithm [23] is employed.

\section{DISCUSSION}

Figure 5 shows the maximum STPV conversion efficiency as a function of $\rho_{\mathrm{BSR}}$, assuming $F_{\mathrm{ec}}=1$ for two cases: a

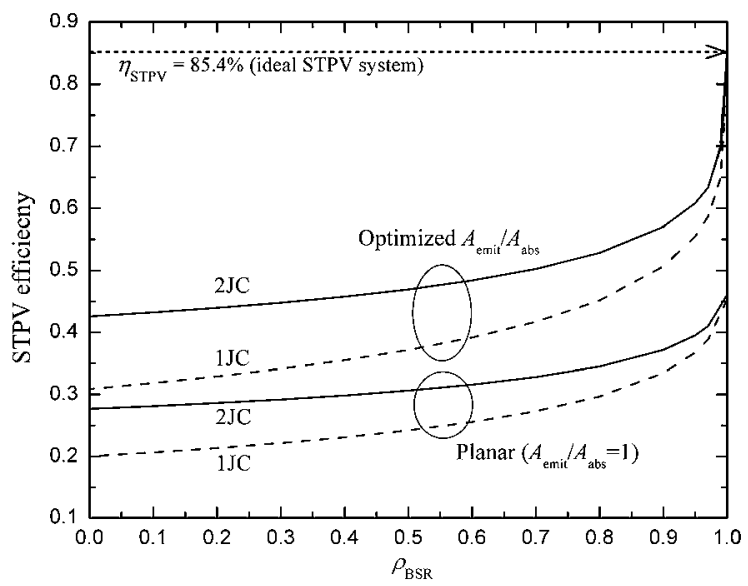

Figure 5. The efficiency limit of solar thermophotovoltaic (STPV) systems using single (1JC) and dual (2JC) junction TPV cells, as a function of the back-side reflector reflectivity $\rho_{\mathrm{BSR}}$, for two

\begin{tabular}{|c|c|c|c|}
\hline Parameter & Symbol & Units & Constraints \\
\hline Sunlight concentration factor & C & - & $1 \leq C \leq C_{\max }$ \\
\hline Absorber cut-off energy & $\varepsilon_{c a}$ & $\mathrm{eV}$ & $\geq 0$ \\
\hline Emitter-to-absorber area ratio & $A_{\text {emit }} / A_{\text {abs }}$ & - & $\geq 1$ \\
\hline Emitter cut-off energy & $\varepsilon_{\mathrm{ce}}$ & $e V$ & $0 \leq \varepsilon_{\mathrm{ce}} \leq \varepsilon_{\mathrm{n}}$ \\
\hline PV cell band-gap(s) & $\varepsilon_{i}(\forall 1 \leq i \leq n)$ & $\mathrm{eV}$ & $\varepsilon_{i} \geq \varepsilon_{i+1}>0$ \\
\hline Cell voltages & $V_{i}(\forall 1 \leq i \leq n)$ & V & $0 \leq V_{i} \leq \varepsilon_{i}$ \\
\hline Reflectivity of the BSR & $\rho_{\mathrm{BSR}}$ & - & $0 \leq \rho_{\mathrm{BSR}} \leq 1$ \\
\hline Emitter-to-cells view factor & $F_{\mathrm{ec}}$ & - & $0 \leq F_{\mathrm{ec}} \leq 1$ \\
\hline Cells-to-cells view factor & $F_{\mathrm{cc}}^{(\mathrm{e})}$ & - & $0 \leq F_{\mathrm{cc}}^{(\mathrm{e})} \leq 1$ \\
\hline Emitter-to-ce $\|$ area ratio & $A_{\text {emit }} / A_{\text {cells }}$ & - & $>0$ \\
\hline
\end{tabular}
cases: a fully optimized STPV system (with an optimized $A_{\text {emit }}$ t $A_{\text {abs }}$ ) and a planar STPV system (with $A_{\text {emit }} / A_{\text {abs }}=1$ ).

Table II. Summary of the parameters used in this paper to model a solar thermophotovoltaic (STPV) system. 


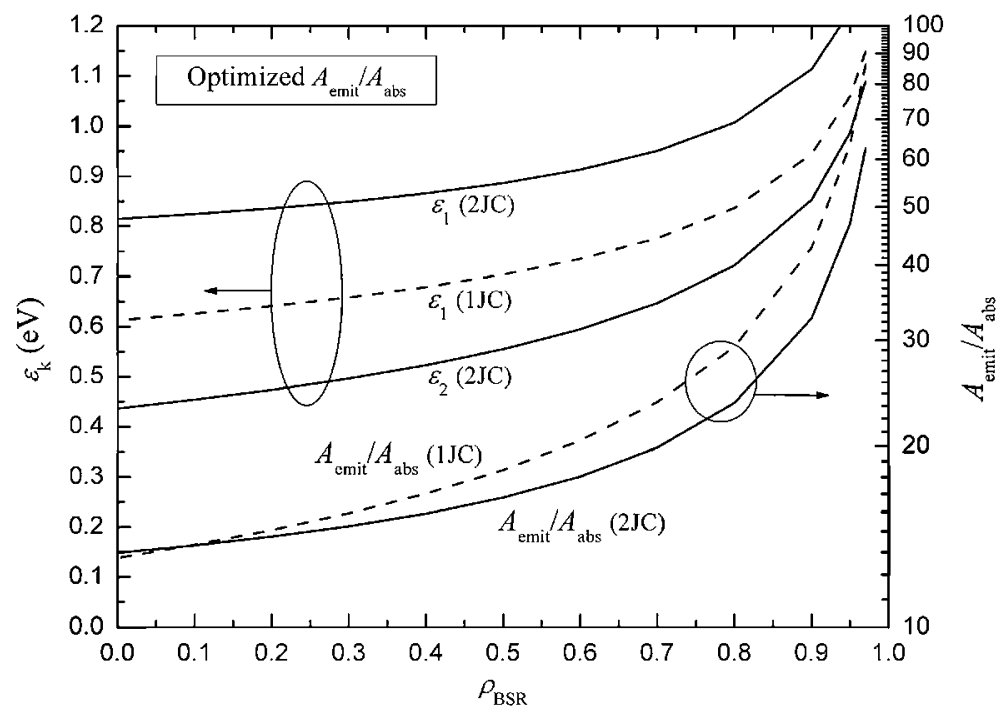

Figure 6. The optimum $A_{\text {emit }} / A_{a b s}$ and TPV cells band-gap energy(ies) (ek) for a fully optimized solar thermophotovoltaic (STPV) system (with an optimized $A_{\text {emit }} / A_{\text {abs }}$ ) using single (1JC) and dual (2JC) junction TPV cells, as a function of the back-side reflector reflectivity $\rho_{\mathrm{BSR}}$. The optimum sunlight concentration factor is $C=C_{\max }=46,050$ and the optimum absorber cut-off energy is $\varepsilon_{\mathrm{ca}}=0 \mathrm{eV}$; they are not shown to simplify the representation.

fully optimized system (with an optimized $A_{\text {emit }} / A_{\text {abs }}$ ) and a planar STPV system (with $A_{\text {emit }} / A_{\text {abs }}=1$ ). Besides, dashed lines represent the case of using single junction TPV cells (1JC) and solid lines represent the case of using dual-junction TPV cells (2JC). Note that for each value of $\rho_{\mathrm{BSR}}$, the rest of system parameters $\left(C, \varepsilon_{\mathrm{ca}}, \varepsilon_{k}\right.$ and $A_{\mathrm{emit}} / A_{\mathrm{abs}}$, when applicable) are optimized. These optimal parameters are shown in Figure 6, for the case of a fully optimized STPV system (with optimized $A_{\text {emit }} / A_{\text {abs }}$ ) and in Figure 7 for the case of an optimized planar STPV system $\left(A_{\text {emit }} / A_{\text {abs }}=1\right)$. In the former case, the optimum $C$ and $\varepsilon_{\mathrm{ca}}$ are $C=C_{\max }=$ 46050 and $\varepsilon_{\mathrm{ca}}=0$ (black-body absorber) for every $\rho_{\mathrm{BSR}}$; they are not shown in Figure 6 to simplify the representation. Finally, Figures 8 and 9 show the electrical power density and the emitter temperature obtained for the fully optimized (with optimized $A_{\text {emit }} / A_{\text {abs }}$ ) STPV system and the optimized planar STPV system, respectively. Table III summarizes all of these results for $\rho_{\mathrm{BSR}}=0.3$ and $\rho_{\mathrm{BSR}}=0.8$.

Note that the maximum STPV conversion efficiency of $85.4 \%$ (Figure 5) is obtained under a set of very restrictive constraints (Figure 6): $\rho_{\mathrm{BSR}}=1, C=C_{\max }=46050, \varepsilon_{\mathrm{ca}}=0$, $\varepsilon_{k} \rightarrow \infty$ and $A_{\text {emit }} / A_{\mathrm{abs}} \rightarrow \infty$. Maximum concentration and a black-body absorber $\left(\varepsilon_{\mathrm{ca}}=0\right)$ are required to minimize the absorber emission losses and to maximize the sunlight absorption, respectively. Besides, ultra-high emitter-to-absorber area ratio and band-gap energy(ies) are required to maximize the energy transfer from the emitter to the cells and to minimize thermalization losses caused by the absorption of photons with higher energies than the cell band-gap, respectively. Note that, in these situations, the ideal STPV system provides the same efficiency and the same emitter temperature (2544K) than the monochromatic STPV system $[2,3]$ and the ideal solar-thermal engine. Unfortunately, none of these requirements are achievable in practice.

\subsection{The relevance of a high emitter-to- absorber area ratio}

Both the emitter-to-absorber area ratio and the band-gap energy(ies) are required to be very high to obtain the highest efficiency (Figure 6). The reason is that, for high band-gap energies and when $\rho_{\mathrm{BSR}} \rightarrow 1$, the radiative exchange between the emitter and the TPV cells becomes almost monochromatic ${ }^{1}$ and consequently, the thermalization losses at the cells are minimized. In this case, the use of a large $A_{\text {emit }} / A_{\text {abs }}$ is required to enhance the energy transfer from the emitter to the TPV cells and therefore compensate the narrow spectral band of the radiative exchange between the cells and the emitter. If $A_{\text {emit }} / A_{\text {abs }}$ would not be high enough, the absorber emission would be too high compared with the emitter emission and consequently, most of the incident energy would be radiated back to the sun.

However, both the optimum emitter-to-absorber area ratio and the band-gap energy(ies) decrease in the presence of cavity losses ${ }^{2}$ (Figure 6). This is because the effect of cavity losses becomes more pronounced when either the band-gap energy(ies) or the emitter-to-absorber area ratio are high. The cavity is responsible for recycling all the photons with energies below the band-gap; therefore, a high

\footnotetext{
${ }^{1} \varepsilon_{1} / T_{\text {emit }}$ is very high, meaning that most of the photons transferred from the emitter to the cells have energies slightly above $\varepsilon_{1}$.

${ }^{2}$ Remember that in this case, the cavity losses consist of optical losses at the BSR of the TPV cells. However, this conclusion still holds for any other kind of cavity losses.
} 


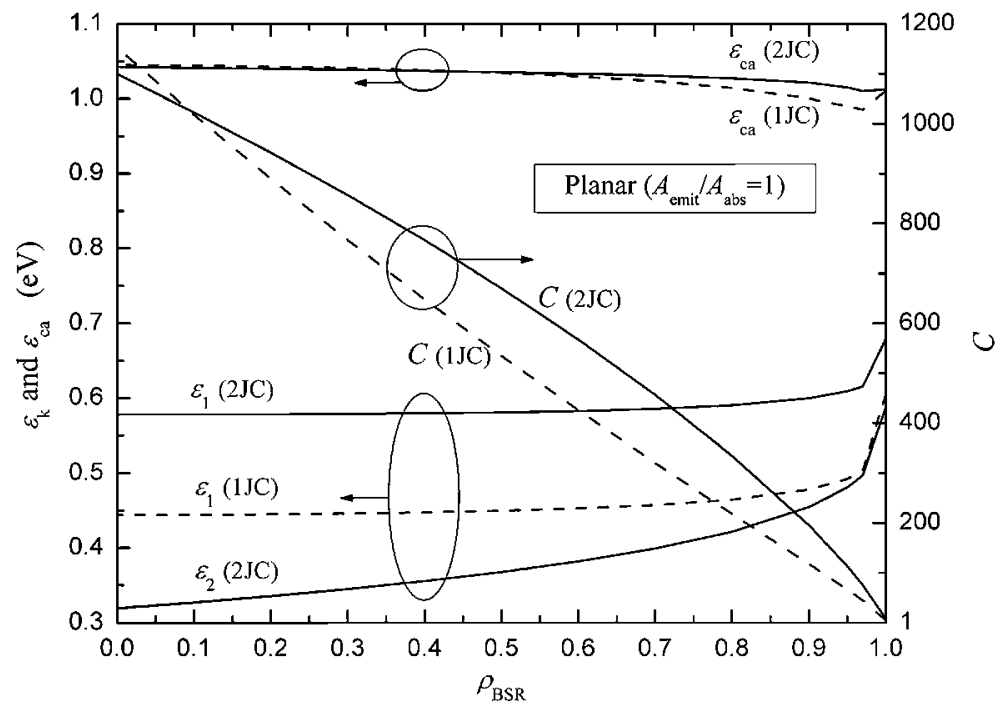

Figure 7. The optimum concentration $(C)$, absorber cut-off energy $\left(\varepsilon_{\mathrm{ca}}\right)$ and thermophotovoltaic (TPV) cells band-gap energy(ies) $\left(\varepsilon_{k}\right)$ for an optimized planar solar TPV system (with $\left.A_{e m i t} / A_{a b s}=1\right)$, using single $(1 \mathrm{JC}$ ) and dual (2JC) junction TPV cells, as a function of the back-side reflector reflectivity $\rho_{\text {BSR }}$.

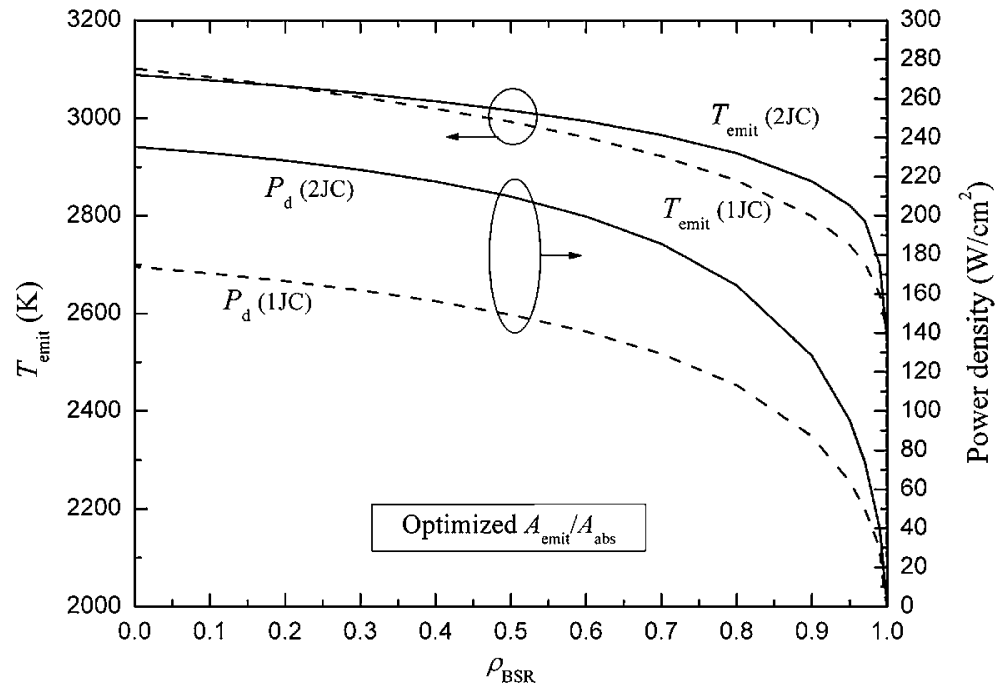

Figure 8. Electrical power density $\left(P_{\mathrm{d}}\right)$ and emitter temperature $\left(T_{\text {emit }}\right)$ for a fully optimized solar thermophotovoltaic (STPV) system (with optimized $A_{\text {emit }} / A_{\text {abs }}$ ), using single $(1 \mathrm{JC})$ and dual (2JC) junction TPV cells, as a function of the back-side reflector reflectivity $\rho_{\mathrm{BSR}}$.

band-gap energy requires a better cavity confinement. Regarding the emitter-to-absorber area ratio, the combination of cavity losses in a system with high emitterto-absorber area ratio would result in a considerably lower emitter temperature, deteriorating the convolution between the emitter spectrum and the spectral response of the TPV cells. Therefore, the optimum emitter-to-absorber area ratio and the corresponding optimum band-gap energy(ies) represent the fulfillment of a trade-off between the maximization of the TPV cells conversion efficiency (minimization of thermalization losses) and the minimization of the impact of the optical cavity losses.

\subsection{Optimization of a planar STPV system}

Despite the fact that very high $A_{\text {emit }} / A_{\text {abs }}$ is required to obtain high system efficiency, a planar STPV system $\left(A_{\text {emit }} / A_{\text {abs }}=1\right)$ offers great practical benefits relative to the TPV optical cavity design, avoiding the use of complex three-dimensional shaped emitter structures [14].

From the previous analysis, we realize that the optimum $A_{\text {emit }} / A_{\text {abs }}$ is greater than one for every $\rho_{\mathrm{BSR}}$ (Figure 6). Therefore, in a planar STPV system, the $A_{\text {emit }} / A_{\text {abs }}$ value is always below the optimum. This means that the absorber emission losses are especially relevant in 


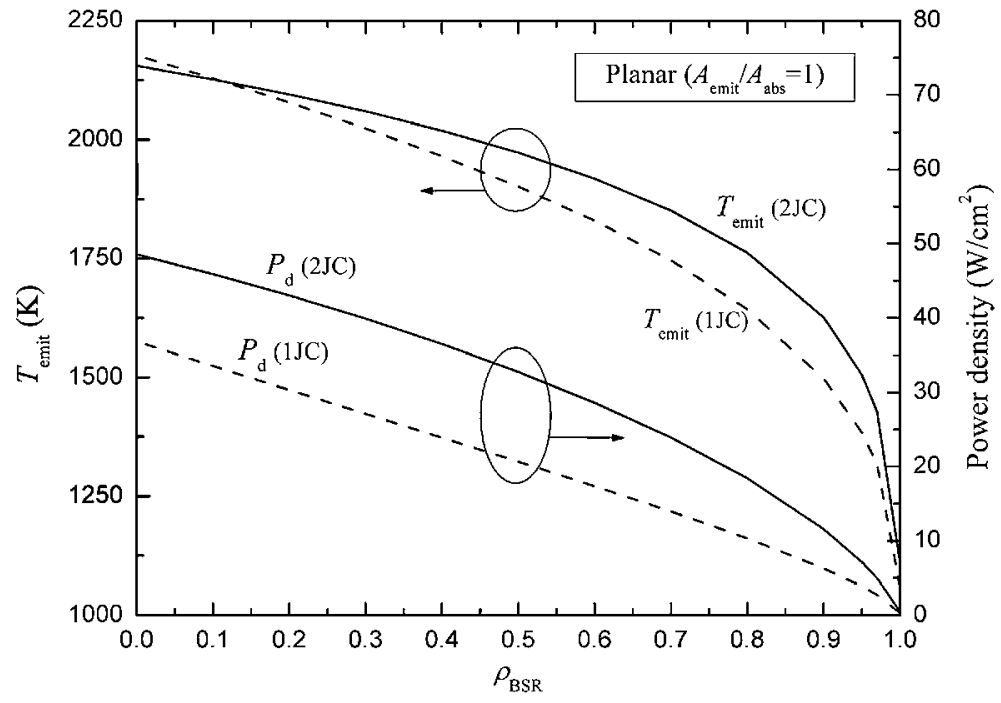

Figure 9. Electrical power density $\left(P_{\mathrm{d}}\right)$ and emitter temperature $\left(T_{\text {emit }}\right)$ for an optimized planar solar thermophotovoltaic (STPV) system (with $A_{\text {emit }} / A_{\mathrm{abs}}=1$ ), using single (1JC) and dual (2JC) junction TPV cells, as a function of the back-side reflector reflectivity $\rho_{\text {BSR }}$.

Table III. Summary of the efficiency limit of different solar thermophotovoltaic (STPV) systems configurations, for the cases $\rho_{\mathrm{BSR}}=0.3$ and $\rho_{\mathrm{BSR}}=0.8$ (with $F_{\mathrm{eC}}=1$ ).

Optimum variables

\begin{tabular}{|c|c|c|c|c|c|c|c|c|c|}
\hline Conf. & $\rho_{\mathrm{BSR}}$ & $\eta_{\text {STPV }}(\%)$ & $P_{\mathrm{d}}\left(\mathrm{W} / \mathrm{cm}^{2}\right)$ & $T_{\text {emit }}(\mathrm{K})$ & $C$ (suns) & $\varepsilon_{\mathrm{Ca}}(\in \mathrm{V})$ & $\varepsilon_{1}(e V)$ & $\varepsilon_{2}(e V)$ & $A_{\text {emit }} / A_{\text {abs }}$ \\
\hline $1 \mathrm{JC}$ & 0.3 & 34.1 & 161.9 & 3043 & $C_{\max }$ & 0 & 0.659 & - & 15.5 \\
\hline Fully optimized & 0.8 & 45.2 & 113.3 & 2872 & $c_{\max }$ & 0 & 0.836 & - & 29.3 \\
\hline $2 \mathrm{JC}$ & 0.3 & 44.8 & 223.5 & 3051 & $c_{\max }$ & 0 & 0.849 & 0.496 & 14.7 \\
\hline Fully optimized & 0.8 & 52.8 & 164.5 & 2928 & $c_{\max }$ & 0 & 1.007 & 0.722 & 23.6 \\
\hline $1 \mathrm{JC}$ & 0.3 & 22.2 & 27.1 & 2023 & 766 & 1.04 & 0.446 & - & 1 (fixed) \\
\hline Planar & 0.8 & 29.7 & 10.3 & 1643 & 218 & 1.01 & 0.465 & - & 1 (fixed) \\
\hline $2 \mathrm{JC}$ & 0.3 & 29.2 & 39.9 & 2059 & 857 & 1.04 & 0.579 & 0.345 & 1 (fixed) \\
\hline Planar & 0.8 & 34.5 & 18.4 & 1762 & 334 & 1.03 & 0.590 & 0.422 & 1 (fixed) \\
\hline
\end{tabular}

these systems. As a consequence, the optimum planar STPV system requires a much lower emitter temperature (Figure 9) than that of a fully optimized system (Figure 8) to avoid excessive absorber emission losses. This is accomplished by means of a lower concentration ratio (much lower than $C_{\max }$, required to fully optimize the STPV system) and the use of a spectrally selective absorber $\left(\varepsilon_{\mathrm{ca}}>0\right)$ (Figure 7$)$. Besides, because of the lower emitter temperature, the band-gap energy(ies) of the TPV cells are also lower than those of the fully optimized system (Figure 7).

Note that the use of a spectrally selective absorber is only advantageous for sufficiently low concentration factors, when the reflection losses in the absorber (photons with energies bellow $\varepsilon_{\mathrm{ca}}$ ) are not as important as its emission losses (photons with energies above $\varepsilon_{\mathrm{ca}}$ ) (Figure 2). In that case, the benefits of the reduction of the absorber emission losses compensate the higher reflection losses, and therefore, the use of a spectrally selective absorber becomes useful.

It must be referred that, in spite of the lower efficiency of planar STPV systems (Figure 5), the less restrictive optimum parameters (specially the concentration ratio and the emitter temperature) and the inherent geometrical simplicity of a planar STPV system make its practical implementation much more affordable.

Table IV summarizes the maximum conversion efficiencies, assuming a lossless TPV cavity $\left(\rho_{\mathrm{BSR}}=1\right.$ and $F_{\text {ec }}=1$ ), for a planar STPV system considering single, dual and triple junction TPV cells. It must be noted that the maximum efficiency of a planar STPV system of $1 \mathrm{JC}$ is $45.3 \%$, exceeding the Shockley-Queisser limit of $40.8 \%$ $[18,19]$. Importantly, this efficiency is obtained under an ultra-low concentration factor of 4.4 suns, in contrast to the Shockley-Queisser limit, which requires the maximum concentration factor. Up to our knowledge, this is 
Table IV. Maximum efficiency of planar solar thermophotovoltaic (STPV) systems $\left(A_{\text {emit }} / A_{\text {abs }}=1\right)$ comprising $\operatorname{single~junction~}(n=1)$ and multijunction (series-connected) TPV cells $(n=2$ and $n=3)$ with an ideal TPV cavity $\left(\boldsymbol{\rho}_{\mathrm{BSR}}=1\right.$ and $\left.F_{\mathrm{ec}}=1\right)$.

\begin{tabular}{ccccccccc} 
& & & \multicolumn{5}{c}{ Optimum variables } \\
\cline { 5 - 9 }$n$ & $\eta_{\text {STPV }}(\%)$ & $P_{\mathrm{d}}\left(\mathrm{W} / \mathrm{cm}^{2}\right)$ & $T_{\text {emit }}(\mathrm{K})$ & $C$ (suns) & $\varepsilon_{\mathrm{Ca}}(\mathrm{eV})$ & $\varepsilon_{1}(\mathrm{eV})$ & $\varepsilon_{2}(\mathrm{eV})$ & $\varepsilon_{3}(\mathrm{eV})$ \\
\hline 1 & 45.3 & 0.32 & 1060 & 4.4 & 1.01 & 0.605 & - & - \\
2 & 46.1 & 0.54 & 1113 & 7.3 & 1.01 & 0.679 & 0.588 & - \\
3 & 47.0 & 0.94 & 1173 & 12.6 & 1.01 & 0.718 & 0.627 & 0.566 \\
\hline
\end{tabular}

the highest efficiency limit reported so far for such low sunlight concentration level and using single junction photovoltaic cells.

\subsection{The impact of the TPV cavity optical losses}

Even minimum presence of optical losses drastically deteriorate the efficiency limit of STPV systems (Figure 5). However, such efficiency barely depends on $\rho_{\mathrm{BSR}}$ for a relatively wide range of $\rho_{\mathrm{BSR}}$ values $\left(\rho_{\mathrm{BSR}}<0.7\right)$. Hence, it becomes necessary to achieve ultra-low optical losses (ultra-high $\rho_{\mathrm{BSR}}$ ) for the photon recycling process to contribute significantly to enhance the efficiency limit of STPV systems. Otherwise, the presence of the photon recycling would barely affect the maximum achievable efficiency. For instance, a system with $\rho_{\mathrm{BSR}}=0.5$ would not provide much higher efficiency limit than that with $\rho_{\mathrm{BSR}}=0$, this is because the full system configuration (sunlight concentration factor, emitter-to-absorber area ratio, band-gap energy of the cells, etc.) can be optimized according to the quantity of optical losses in the cavity, minimizing their impact. As such, and perhaps contrary to intuition, the optimization of the full system configuration has to be considered as a priority action for practical STPV systems rather than the minimization of the optical losses in the photon recycling system. ${ }^{3}$

For the same reason, any improvement in the photon recirculation system (increasing $\rho_{\mathrm{BSR}}$ ) must be accompanied by a re-optimization of the system parameters, to ensure that such improvement significantly increases the efficiency limit of the resulting system. In a planar STPV system (Figure 7), such re-optimization mainly consists in modifying the concentration factor. However, in a fully optimized STPV system (Figure 6), it would also be required to recalculate the optimum emitter-to-absorber area ratio and the band-gap energy(ies) of the cells.

\footnotetext{
${ }^{3}$ This analysis assumes $F_{\mathrm{ec}}=1$, so that all the photons originating at the emitter impinge the cells. It is important to note that the cavity losses represented by $F_{\mathrm{ec}}<1$ will have a stronger impact on the system efficiency (note that $F_{\mathrm{ec}}$ is squared in Equation (20)).
}

\subsection{The relevance of using multijunction TPV cells}

There are two main benefits of using MJCs: First, they attenuate the impact of the cavity losses $\left(\rho_{\mathrm{BSR}}<1\right)$ on the system efficiency limit (Figure 5). Second, they provide a notably higher electrical power density than single junction TPV cells (1JC) (Figures 8 and 9).

In the case of $1 \mathrm{JC}$, a significant part of the emitter spectrum is not properly used by the cells. Consequently, the photon recycling process, which turns back to the emitter the sub-band-gap photons, becomes especially relevant. MJCs allow for a more efficient direct conversion of the emitter radiation into electricity. Therefore, the use of a photon recycling system is not as important. In fact, the use of MJCs could result in a more efficient approach, to enhance both the conversion efficiency and the electrical power density of STPV systems than the incorporation of a photon recycling system. For instance, a planar STPV system with $\rho_{\mathrm{BSR}}=0$ comprising dualjunction TPV cells (2JC) shows an efficiency limit of $27.7 \%$ (Figure 5 ). The same system with single junction TPV cells would require $\rho_{\mathrm{BSR}} \approx 0.7$ to obtain the same efficiency limit. Moreover, the electrical power density obtained in the first case (2JC and $\rho_{\mathrm{BSR}}=0$ ) would be $48.5 \mathrm{~W} / \mathrm{cm}^{2}$ (Figure 9) whereas in the second case (1JC and $\rho_{\mathrm{BSR}}=0.7$ ) would be $13.9 \mathrm{~W} / \mathrm{cm}^{2}$, because of the considerably higher concentration ratio required in the first case (Figure 7).

Besides, for a given $\rho_{\mathrm{BSR}}$, STPV systems comprising MJCs can output a higher electrical power density than that of 1JC (Figures 8 and 9). Importantly, such higher electrical power density is obtained without requiring a much higher emitter temperature.

For $\rho_{\mathrm{BSR}}>0.1$, the slightly higher emitter temperature obtained for 2JC than for 1JC (Figures 8 and 9) is attributed to the lower optimum $A_{\text {emil }} / A_{\text {abs }}$ (in the case of a fully optimized STPV system, Figure 6) or to the higher optimum concentration factor (in the case of a planar STPV system, Figure 7). Higher emitter temperature is possible when using 2JC because the improvement of the energy transfer from the emitter to the cells (reduction of the cavity losses) compensates the slightly higher absorber emission losses related with the higher absorber/emitter temperature.

In contrast, when $\rho_{\mathrm{BSR}}<0.1$, the STPV system using $1 \mathrm{JC}$ requires a slightly higher emitter temperature than the one using 2JC (Figures 8 and 9). This is attributed to the 
lower optimum $A_{\text {emil }} / A_{\text {abs }}$ (in the case of a fully optimized STPV system, Figure 6) or to the higher optimum concentration factor (in the case of a planar STPV system, Figure 7). Higher emitter temperature is required for $1 \mathrm{JC}$ when $\rho_{\mathrm{BSR}}<0.1$ to minimize the impact of the very high cavity losses, because the higher emitter temperature allows to enhance the spectral convolution between the $1 \mathrm{JC}$ spectral response and the emitter spectrum.

Finally, taken into account the optimum band-gaps for the 2JC device (Figures 6 and 7 and Table III), we must note that the InGaAsSb quaternary compounds, grown on GaSb substrates, are good candidates to manufacture 2JC for STPV applications. In this case, the very thin InGaAsSb active layers should be transferred to a highly reflective substrate to avoid undesirable absorption in the GaSb substrate.

\subsection{The relevance of using spectrally selective absorbers}

In the previous analysis concerning planar STPV systems, it was seen that the optimization of this kind of systems requires a relatively low concentration factor (much lower than the maximum) and the use of a spectrally selective absorber (Figure 7). Both aspects are necessary to minimize the absorber emission losses, which are particularly relevant in planar STPV systems. On the other hand, in the case of a fully optimized STPV system (with optimized $A_{\text {emit }} / A_{\text {abs }}$ ), a black-body absorber and the maximum concentration ratio are required. To analyze all the intermediate cases between both optimized systems, in this section, we present the optimization of STPV systems with any combination of $C$ and $A_{\text {emit }} / A_{\text {abs }}$.

For that, we decided to focus only on single junction TPV cells, for the sake of simplicity. Nevertheless, the same qualitative conclusions are valid for MJCs. We performed the optimization of two kinds of STPV systems: One with an optimized $\varepsilon_{\mathrm{ca}}$ and the other with a black-body absorber $\left(\varepsilon_{\mathrm{ca}}=0\right)$. These systems were optimized over a broad range of concentration factors and emitter-to-absorber area ratios, assuming $10 \%$ of cavity losses $\left(F_{\mathrm{ec}}=0.9\right)$ and an ideal BSR $\left(\rho_{\mathrm{BSR}}=1\right)$. The optimization parameters were the following: the band-gap energy of the TPV cells $\left(\varepsilon_{1}\right)$ and (when applicable) the absorber cut-off energy $\left(\varepsilon_{\mathrm{ca}}\right)$.

Figures 10-13 show, respectively, the optimum $\varepsilon_{\mathrm{ca}}$, the optimum band-gap energy $\left(\varepsilon_{1}\right)$, the resultant emitter temperature and the electrical power density of the STPV system with an optimized $\varepsilon_{\text {ca. }}$. Figures 14-16 show the optimum band-gap energy, the emitter temperature and the electrical power density of the STPV system with a blackbody absorber. In all these figures, the STPV conversion efficiency is superimposed.

We corroborate that the use of a spectrally selective absorber is required in a broad range of cases, to optimize STPV systems with relatively low sunlight concentration factor and emitter-to-absorber area ratios (Figure 10), which are common characteristics in most practical cases.

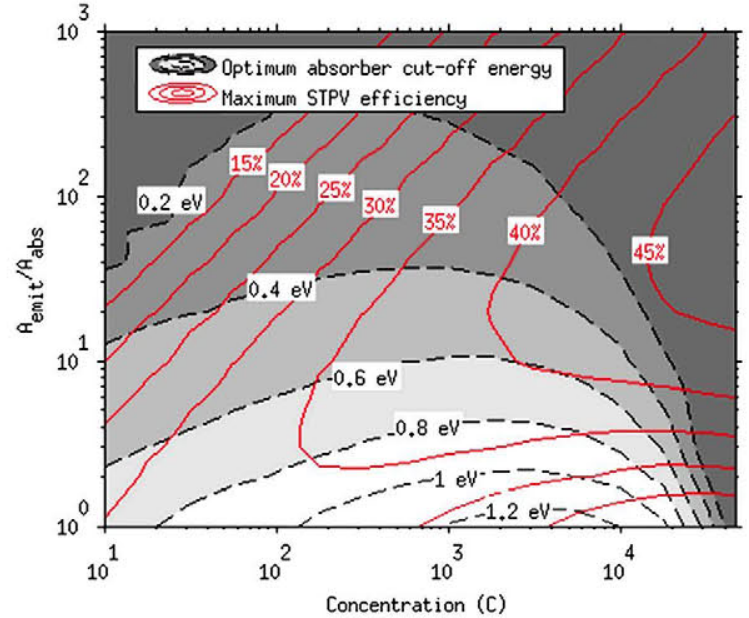

Figure 10. Contour-plot of the optimum absorber cut-off energy $\left(\varepsilon_{\mathrm{ca}}\right)$ with superimposed iso-solar thermophotovoltaic (STPV) efficiency ( $\eta_{\text {STPV }}$ ) curves (solid lines), as a function of the concentration ( $C$ ) and the emitter-to-absorber area ratio $\left(A_{\text {emit }} / A_{\text {abs }}\right)$. This simulation uses an optimized spectrally selective absorber, single junction TPV cells and $F_{\mathrm{ec}}=0.9$ (i.e., $10 \%$ of cavity losses).

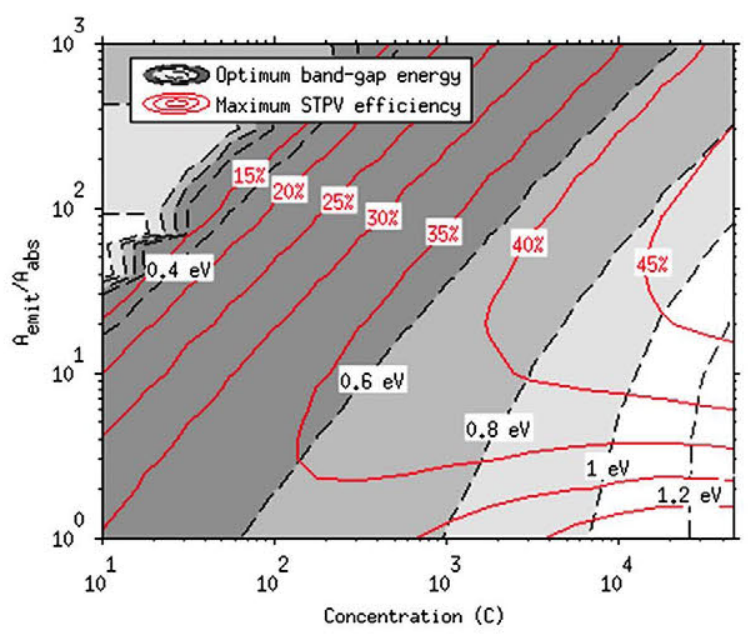

Figure 11. Contour-plot of the optimum thermophotovoltaic (TPV) cell band-gap energy $\left(\varepsilon_{\text {gap }}\right)$ with superimposed iso-solar TPV (STPV) efficiency ( $\eta_{\text {STPV }}$ ) curves (solid lines), as a function of the concentration $(C)$ and the emitter-to-absorber area ratio $\left(A_{\text {emit }} / A_{\text {abs }}\right)$. This simulation uses an optimized spectrally selective absorber, single junction TPV cells and $F_{\text {ec }}=0.9$ (i.e., $10 \%$ of cavity losses).

With spectrally selective absorbers, the efficiency limit of STPV systems, assuming $10 \%$ of cavity losses and single junction TPV cells, could be in the range of $35 \%(C$ 120 suns, $T_{\mathrm{emit}} \approx 1400 \mathrm{~K}, A_{\mathrm{emit}} / A_{\mathrm{abs}} \approx 3$ and $P_{\mathrm{d}} \approx 5 \mathrm{~W} /$ $\left.\mathrm{cm}^{2}\right)$ to $40 \%\left(C \approx 1000\right.$ suns, $T_{\text {emit }} \approx 1600 \mathrm{~K}, A_{\text {emit }} / A_{\text {abs }}$ 10 and $P_{\mathrm{d}} \approx 10 \mathrm{~W} / \mathrm{cm}^{2}$ ). Moreover, the electrical power density can be increased at the expense of a lower conversion efficiency by reducing the emitter-to-absorber area ratio. For instance, conversion efficiencies of about $30 \%$ are feasible with electrical power densities above 


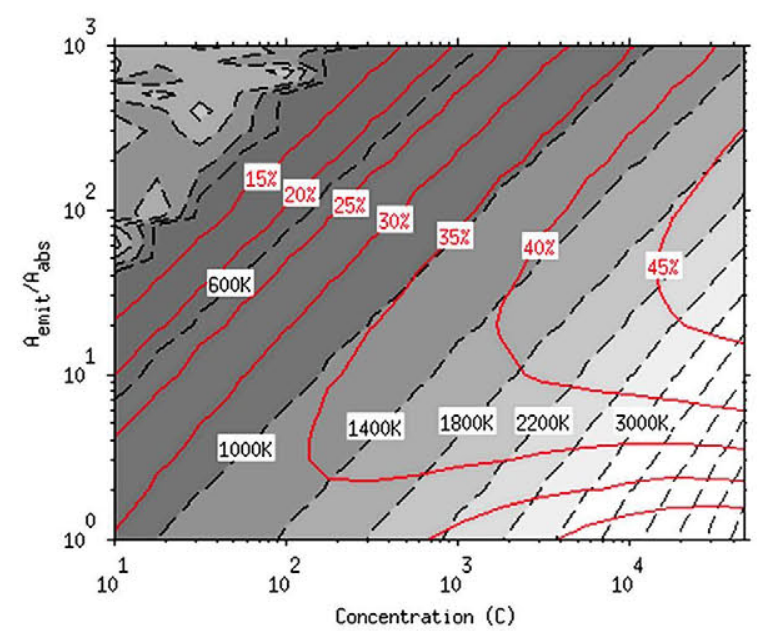

Figure 12. Contour-plot of the emitter temperature $\left(T_{\text {emit }}\right)$ with superimposed iso-solar thermophotovoltaic (STPV) efficiency ( $\left.\eta_{\mathrm{STPV}}\right)$ curves (solid lines), as a function of the concentration $(C)$ and the emitter-to-absorber area ratio $\left(A_{\text {emit }} / A_{\text {abs }}\right)$. This simulation uses an optimized spectrally selective absorber, single junction TPV cells and $F_{\text {ec }}=0.9$ (i.e., $10 \%$ of cavity losses).

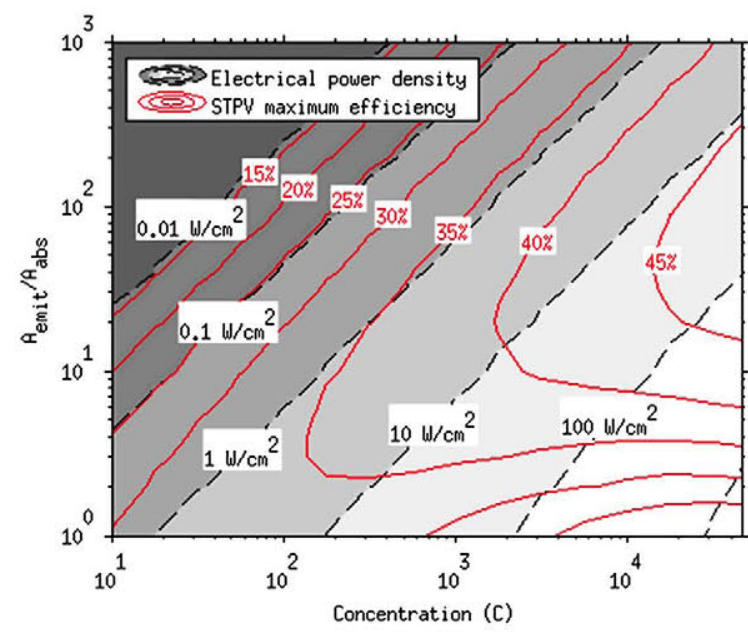

Figure 13. Contour-plot of the electrical power density $\left(P_{\mathrm{d}}\right)$ with superimposed iso-solar thermophotovoltaic (STPV) efficiency ( $\eta_{\mathrm{STPV}}$ ) curves (solid lines), as a function of the concentration (C) and the emitter-to-absorber area ratio $\left(A_{\text {emit }} / A_{\text {abs }}\right)$. This simulation uses an optimized spectrally selective absorber, single junction TPV cells and $F_{\text {ec }}=0.9$ (i.e., $10 \%$ of cavity losses).

$50 \mathrm{~W} / \mathrm{cm}^{2}$ (Figure 13) if the emitter-to-absorber area ratio is reduced from the optimum (in the range of 10-50) down to $2-3$, while keeping a high concentration factor (slightly above 1000 suns). However, in this case, very high emitter temperature is required (above $2200 \mathrm{~K}$ ). High electrical power density is important to reduce the cost of the produced energy.

It is also important to note that, if a black-body absorber is used instead of a spectrally selective absorber, the combination of high concentration and high emitterto-absorber area ratio is particularly relevant. For instance,

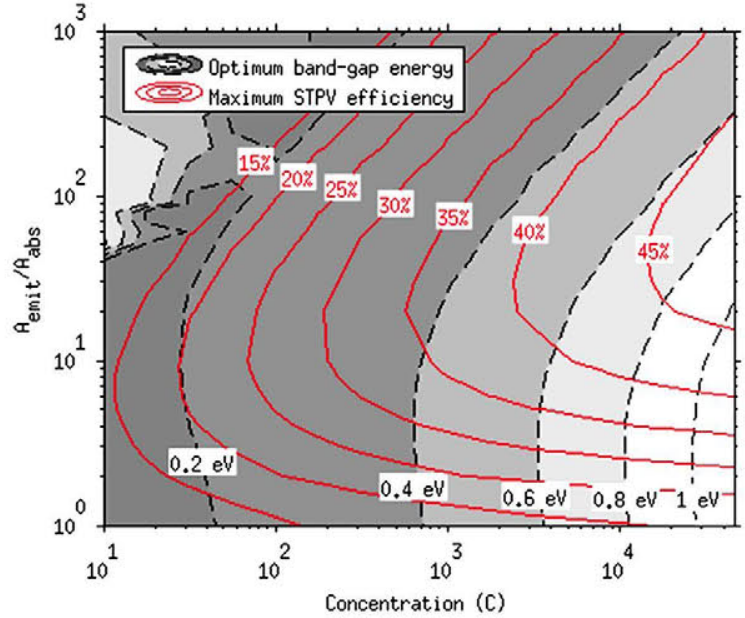

Figure 14. Contour-plot of the optimum band-gap energy ( $\left.\varepsilon_{\text {gap }}\right)$ with superimposed iso-solar thermophotovoltaic (STPV) efficiency ( $\eta_{\text {STPV }}$ ) curves (solid lines), as a function of the concentration $(C)$ and the emitter-to-absorber area ratio $\left(A_{\text {emil }} / A_{\text {abs }}\right)$. This simulation uses a black-body absorber $\left(\varepsilon_{\mathrm{ca}}=0 \mathrm{eV}\right)$, single junc-

tion TPV cells and Fec $=0.9$ (i.e., $10 \%$ of cavity losses).

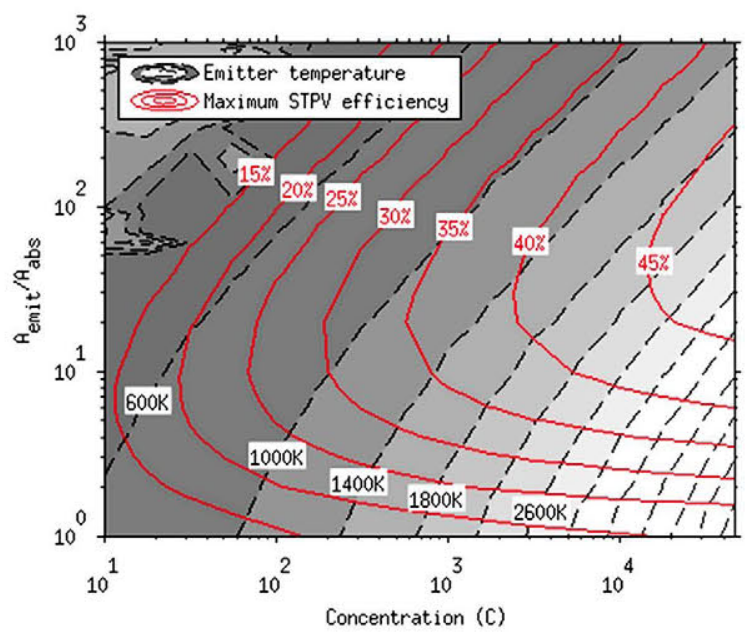

Figure 15. Contour-plot of the emitter temperature $\left(T_{\text {emit }}\right)$ with superimposed iso-solar thermophotovoltaic (STPV) efficiency $\left(\eta_{\text {STPV }}\right)$ curves (solid lines), as a function of the concentration (C) and the emitter-to-absorber area ratio $\left(A_{\text {emit }} / A_{\text {abs }}\right)$. This simulation uses a black-body absorber $\left(\varepsilon_{\mathrm{ca}}=0 \mathrm{eV}\right)$, single junction TPV cells and $F_{\mathrm{ec}}=0.9$ (i.e., $10 \%$ of cavity losses).

with an optimized spectrally selective absorber, a combination of $C=120$ suns and $A_{\text {emil }} / A_{\text {abs }}=3$ is enough to provide an efficiency of $35 \%$ (Figures 10-13). In contrast, if a black-body absorber were used instead, it would be required about $C=800$ and $A_{\text {emil }} / A_{\text {abs }}=10$ to achieve the same efficiency (Figures 14-16). Finally, another consequence of using black-body absorbers is that the optimum band-gap energy is slightly lower (Figures 11 and 14). This is due to the slightly lower resultant emitter temperature, which can be realized by comparing Figures 12 and 15 . 


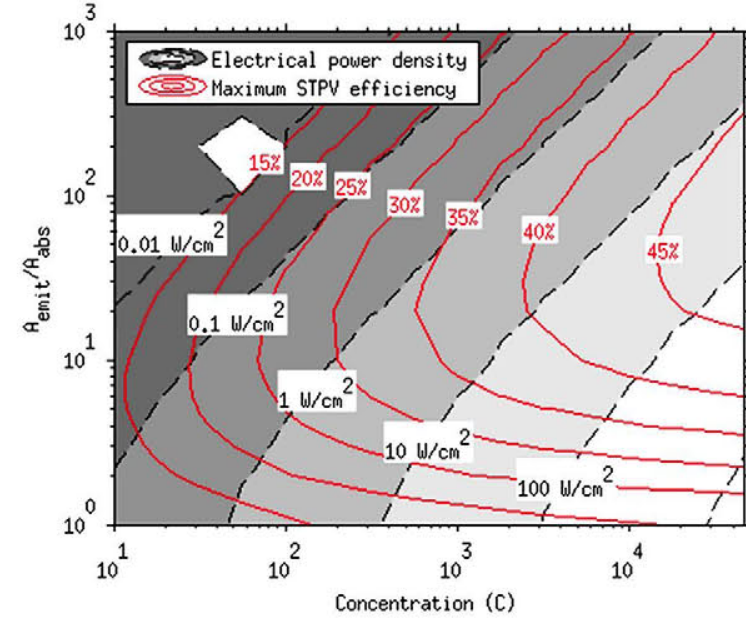

Figure 16. Contour-plot of the electrical power density $\left(P_{\mathrm{d}}\right)$ with superimposed iso-solar thermophotovoltaic (STPV) efficiency $\left(\eta_{\mathrm{STPV}}\right)$ curves (solid lines), as a function of the concentration $(C)$ and the emitter-to-absorber area ratio $\left(A_{\text {emit }} / A_{\text {abs }}\right)$. This simulation uses a black-body absorber $\left(\varepsilon_{c a}=0 \mathrm{eV}\right)$, single junction TPV cells and $F_{\mathrm{ec}}=0.9$ (i.e., $10 \%$ of cavity losses).

\subsection{The relevance of very high emissivity}

We remind to the reader that all the results presented in this paper assume an emissivity of one. In the case of a fully optimized STPV system, a lower emitter emissivity would result in a higher optimum $A_{\text {emit }} / A_{\text {abs }}$. This is due to the fact that the total irradiance leaving the emitter would be given by the product of the emitter area times the emissivity, rather than by the emitter area alone. Therefore, the optimum emitter area should increase to compensate the lower emitter emissivity. In addition, the system would become more sensitive to the cavity losses, because they are more relevant for high $A_{\text {emit }} / A_{\text {abs }}$. This points out the importance of high emitter emissivity for STPV systems.

In the case of a planar STPV system, for which $A_{\text {emit }} / A_{\text {abs }}$ is fixed to one, the lower emitter emissivity inevitably results in excessive absorber emission losses, deteriorating the conversion efficiency. Therefore, a high emitter emissivity is particularly important for planar STPV systems to maximize the energy transfer from the emitter to the cells. Other alternatives to enhance the energy transfer from the emitter to the cells (especially relevant for planar STPV systems) are given in the next section.

\section{ALTERNATIVES TO HIGH EMITTER-TO-ABSORBER AREA RATIOS}

In the previous analysis, we have pointed out the great relevance of high emitter-to-absorber area ratios $\left(A_{\text {emit }} / A_{\text {abs }}\right)$ to obtain high STPV conversion efficiency. High emitterto-absorber area ratio is required to enhance the energy transfer from the emitter to the cells and consequently, to minimize the impact of the absorber emission losses.

In this paper, we have assumed diffuse emitters and absorbers surrounded by air, which made the étendue $H$ (Equation 6) to be simply formulated as $H_{\text {emit }}=\pi A_{\text {emit }}$ and $H_{\text {abs }}=\pi A_{\text {abs }}$, respectively (Table I). In this case, the only alternative to enhance the energy transfer from the emitter to the cells (i.e., the absorber-to-emitter étendue ratio) is by increasing $A_{\text {emit }} / A_{\text {abs }}$. However, in the most general case, the étendue is not only a function of the area but also of the refractive index of the medium surrounding the emitting surface and of the angle of the emission (Equation 6). It leads to additional alternatives to enhance the energy transfer.

In this regard, three more alternatives have been proposed so far to enhance the absorber-to-emitter étendue ratio: the micron-gap TPV (MTPV) concept [24,25], the light-pipe TPV (LTPV) concept [26] and the use of angle-selective absorbers $[27,28]$. They are summarized in Table V. Both MTPV and LTPV concepts aim to enhance the number of propagating modes from the emitter to the cells. The MTPV concept $[24,25]$ consists of reducing the emitter-to-cells distance to the nano/micro-metric scale, allowing the propagation of the evanescent modes. The LTPV concept [26] consists of filling the volume between the emitter and the cells with a low thermally conductive and transparent material with a high refraction index. In contrast to the high emitter-to-absorber area approach, both MTPV and LTPV allow the enhancement of the energy transfer without deteriorating the electrical power density. Unfortunately, in both cases, the maximum achievable absorber-to-emitter étendue ratio is limited to $n^{2}$, being $n$ the density of photonic states that are transmitted from the emitter to the cells for the MTPV concept, or the refraction index of the medium in between the emitter and the cells for the LTPV concept. It makes difficult to exceed an energy transfer gain of 10 , approximately.

In the case of angle-selective absorbers [27,28], the emission/absorption at the absorber surface is restricted to a certain cone of angles. In contrast to the previous alternatives, this approach shows an additional attractiveness

Table V. Strategies to enhance the energy transfer from the emitter to the cells, that is, to achieve a high $H_{\text {emit }} / H_{\text {abs }}$.

\begin{tabular}{lll}
\hline Concept & Actuating parameter & Maximum $H_{\text {emit }} / H_{\text {abs }}$ \\
\hline Micron-gap thermophotovoltaic (MTPV) & $n_{\text {out }}$ & up to $\approx 10$ \\
Light-pipe TPV (LTPV) & $n_{\text {out }}$ & up to $\approx 10$ \\
Angle-selective absorber & $\theta_{\text {abs }}$ & up to $1 / \sin ^{2} \theta_{\text {sun }} \approx 46,000$ \\
Large emitter-to-absorber area ratio & $A_{\text {emit }} / A_{\text {abs }}$ & $\rightarrow \infty$ \\
\hline
\end{tabular}


(at least from the theoretical point of view) because they could restrict the radiative exchange of the absorber to the sun disc only, without the necessity of a maximum sunlight concentration optical system. Besides, the absorberto-emitter étendue ratio can be very large (up to 46,000) while keeping a relatively high electrical power density (depending on the concentration factor).

Note that all these alternatives could be combined in the same STPV design. It could provide a very high energy transfer without the necessity of the very complicated three-dimensional shape STPV configurations (typically cylindrical) required to enhance the emitter-to-absorber area ratio.

\section{SUMMARY AND CONCLUSIONS}

In this paper, we have presented a global analysis of an STPV system. The analysis has consisted of a multivariable optimization of the system, which has been modeled according to the detailed balance theory. The system considered comprises a sunlight concentrator system, a spectrally selective absorber and multijunction TPV cells with back-side reflectors. We summarize some of the main conclusions:

- The highest efficiency limit (of $85.4 \%$ ) is obtained for a system without optical losses, operated under the maximum sunlight concentration factor (about 46,000 suns) and for which the emitter-to-absorber area ratio and the band-gap energy of the TPV cells, both tends to infinity. In this situation, the electrical power density tends to zero, which means that the required system would be huge. Besides, a minimal presence of optical losses in the TPV cavity drastically deteriorates that efficiency. These are the reasons why an efficiency of $85.4 \%$, despite being the limiting one, is unachievable by practical systems.

- Ultra-low optical losses are required to obtain notable benefits from the photon recycling system on the efficiency limit of STPV systems. Otherwise, the presence of optical losses barely affects to the maximum achievable efficiency. It is because the full system configuration can be optimized, depending on the quantity of optical losses in the cavity, minimizing the impact of the cavity losses. As a consequence, the optimization of the full system configuration (concentration ratio, emitter-to-absorber area ratio, TPV cells band-gap energies, etc.) must be considered as a priority action line, which is, at least, as important as the improvement of the photon recycling system.

- The use of multijunction TPV cells (rather than single junction TPV cells) presents two key benefits: First, they notably attenuate the impact of the cavity losses on the system efficiency limit. Second, they provide a notably higher electrical power density without the necessity of a much higher emitter temperature.
- The use of an optimized spectrally selective absorber result in higher efficient practical STPV designs, which are characterized by a concentration factor much lower than the maximum and a low emitter-to-absorber area ratio (for instance, a planar STPV system). On the other hand, if a black-body absorber is used, the combination of high emitter-to-absorber area ratio and an ultra-high concentration ratio are particularly relevant to enhance the conversion efficiency.

- For practical systems (assuming 10\% of optical losses in the TPV cavity, sunlight concentration ratio of about 1000-2000 suns and emitter-to absorber area ratio of about 2-3) ultra-high electrical power densities (about $50 \mathrm{~W} / \mathrm{cm}^{2}$ ) are achievable with conversion efficiencies exceeding $30 \%$, using single junction TPV cells.

\section{ACKNOWLEDGEMENTS}

The authors gratefully acknowledge A. Martí and E. Antolín for the fruitful discussions about the basic operation principles of photovoltaic cells, to P. Benítez for answering several doubts concerning the optical concepts dealt in the paper and to P. Espinet and G. del Coso for their valuable support to configure the simulation workstations. A. Datas also acknowledges financial support from the "Consejería de Educación de la Comunidad de Madrid y del Fondo Social Europeo (FSE)".

\section{REFERENCES}

1. Swanson R. A proposed thermophotovoltaic solar energy conversion system. Proceedings of the IEEE 1979; 67: 446-447.

2. Luque A, Hegedus S. Handbook of Photovoltaic Science and Engineering. Wiley: Chichester, 2003.

3. Davies P, Luque A. Solar thermophotovoltaics-brief review and new look. Solar Energy Materials and Solar Cells 1994; 37(1): 11-22.

4. Chubb D, Good B, Lowe R. Solar thermophotovoltaic (stpv) system with thermal energy storage. In $2 n d$ NREL Conference on Thermophotovoltaic Generation of Electricity, no. 358, 1995, pp. 181-198.

5. Stone K, Fatemi N, Garverick L. Operation and component testing of a solar thermophotovoltaic power system. In 25th IEEE Photovoltaic Specialists Conference, 1996, pp. 1421-1424.

6 . Bell R. Concentration ratio and efficiency in thermophotovoltaics. Solar Energy 1979; 23(3): 203-210.

7. Demichelis F, Minetti-Mezzetti E. A solar thermophotovoltaic converter. Solar Cells 1980; 1(4): 395- 403.

8. Edenburn M. Analytical evaluation of a solar thermophotovoltaic (tpv) converter. Solar Energy 1980 24(4): 367-371. 
9. Höfler H, Paul H, Ruppel W, Würfel P. Interference filters for thermophotovoltaic solar energy conversion. Solar Cells 1983; 10: 273-286.

10. Höfler H, Würfel P, Ruppel W. Selective emitters for thermophotovoltaic solar energy conversion. Solar Cells 1983; 10: 257-271.

11. Spirkl W, Ries H. Solar thermophotovoltaics: an assessment. Journal of Applied Physics 1985; 57(9): 4409-4414.

12. Würfel P, Ruppel W. Upper limit of thermophotovoltaic solar-energy conversion. IEEE Transactions on Electron Devices 1980; 27: 745-750.

13. Chaudhuri T. A solar thermophotovoltaic converter using Pbs photovoltaic cells. International Journal of Energy Research 1992; 16(16): 481-487.

14. Harder N-P, Würfel P. Theoretical limits of thermophotovoltaic solar energy conversion. Semiconductor Science and Technology 2003; 18: S151-S157.

15. Andreev V, Khvostikov V, Khvostikova O, Rumyantsev V, Gazarjan P, Vlasov A. Solar thermophotovoltaic converters: efficiency potentialities. In Sixth Conference on Thermophotovoltaic Generation of Electricity, Vol. 738, Gopinath JLA, Coutts TJ (eds). AIP Conference Proceedings: Freiburg, Germany2004; 96-104.

16. Datas A, Algora C. Analytical model of solar thermophotovoltaic systems with cylindrical symmetry: ray tracing approach. Progress in Photovoltaics 2009; 17: $526-541$.

17. Datas A, Algora C. Detailed balance analysis of solar thermophotovoltaic systems made up of single junction photovoltaic cells and broadband thermal emitters. Solar Energy Materials and Solar Cells 2010; 94(12): 2137-2147.

18. Shockley W, Queisser H. Detailed balance limit of efficiency of $\mathrm{p}-\mathrm{n}$ junction solar cells. Journal of Applied Physics 1961; 32: 510-519.
19. Araújo G, Martí A. Absolute limiting efficiencies for photovoltaic energy conversion. Solar Energy Materials and Solar Cells 1994; 33: 213-240.

20. Siegel R, Howell J. Thermal Radiation Heat Transfer. Mc Graw Hill: Chichester, 1972.

21. Würfel P. The chemical potential of radiation. Journal of Physics C: Solid State Physics 1982; 15: 3967-3985.

22. Winston R, Miñano J, Benitez P. Nonimaging Optics. Elsevier: London, 2005.

23. Lagarias J, Reeds J, Wright M, Wright P. Convergence properties of the Nelder-Mead simplex method in low dimensions. SIAM Journal on Optimization 1998; 9(1): 112-147.

24. DiMatteo R, Greiff P, Finberg S, Young-Waithe K, Choy H, Masaki M, Fonstand C. Enhanced photogeneration of carriers in a semiconductor via coupling across a nonisothermal nanoscale vacuum gap. Applied Physics Letters 2001; 79(12): 1894-1896.

25. Pan J, Choy H, Fonstad C. Very large radiative transfer over small distances from a black body for thermophotovoltaic applications. IEEE Transactions on Electron Devices 2000; 47(1): 241-249.

26. Chubb DL. Light pipe thermophotovoltaics (LTPV). In Thermophotovoltaic Generation of Electricity, 2007, Proceedings Paper.

27. Florescu M, Lee H, Puscasu I, Pralle M, Florescu L, Ting D, Dowling J. Improving solar cell efficiency using photonic band-gap materials. Solar Energy Materials and Solar Cells 2007; 91: 1599-1610.

28. Datas A, Celanovic I, Algora C. Efficiency limit of planar geometry solar thermophotovoltaic systems using angular selective absorbers. In 9th World Conference on thermophotovoltaic generation of electricity-25th European Photovoltaic Solar Energy Conference and Exhibition-5th World Conference on Photovoltaic Energy Conversion, 2010. 